\title{
Strategieentwicklung in Krisenzeiten
}

\begin{abstract}
„Von Managern wird jetzt Klarheit erwartet in einer Situation, die nicht selbst beherrschbar ist und deren Auswirkungen man auch noch nicht abschätzen kann. Das erfordert wahres Leadership. Also nicht nur das risikoaverse Management des Status Quo. Führungskräfte müssen vielmehr in dieser Situation hoher Ungewissheit Verantwortung übernehmen und Risiken eingehen. Leadership steht dabei auch für die Übernahme gesellschaftlicher Verantwortung.“
\end{abstract}

Dirk von Manikowsky, Partner Hering Schuppener, März 2020

In jeder Krise werden Unternehmen von einem Moment auf den anderen vor kurzfristige, überlebenskritische Entscheidungen gestellt. Gleichzeitig muss die mittel- und langfristige Strategie in Rekordzeit an die neuen Gegebenheiten angepasst werden, um nicht nur erfolgreich durch, sondern auch gestärkt aus der Krise zu kommen.

Im vorigen Kapitel wurde bereits dargelegt, warum eine proaktive Strategie mit passenden defensiven und vor allem auch offensiven Maßnahmen in Krisenzeiten entscheidend ist. Es mag auf den ersten Blick kontraproduktiv erscheinen, in einer hochdynamischen Krisensituation strategisch zu planen statt „erst einmal zu machen“. Doch auch hier zeigt ein Blick auf die Krisen der Vergangenheit, dass ein reflektiertes Vorgehen einem reflexartigen Aktionismus klar vorzuziehen ist. So wurden beispielsweise in einer Studie der „Association for Strategic Planning“ die strategischen Planungsaktivitäten von knapp 200 US-Unternehmen während der Finanzkrise untersucht. Am erfolgreichsten waren laut der Studie diejenigen Unternehmen, die besonders ausgeprägte und effektive strategische Planungsaktivitäten vorweisen konnten. Diese Unternehmen waren nicht nur in finanzieller Hinsicht erfolgreicher. Sie konnten auch eine deutlich stärkere Proaktivität vorweisen, hatten das Gefühl, gut auf den Wandel vorbereitet zu sein und zeigten einen stärkeren kurzfristigen Optimismus (Abb. 2.1). Bemerkenswert ist 


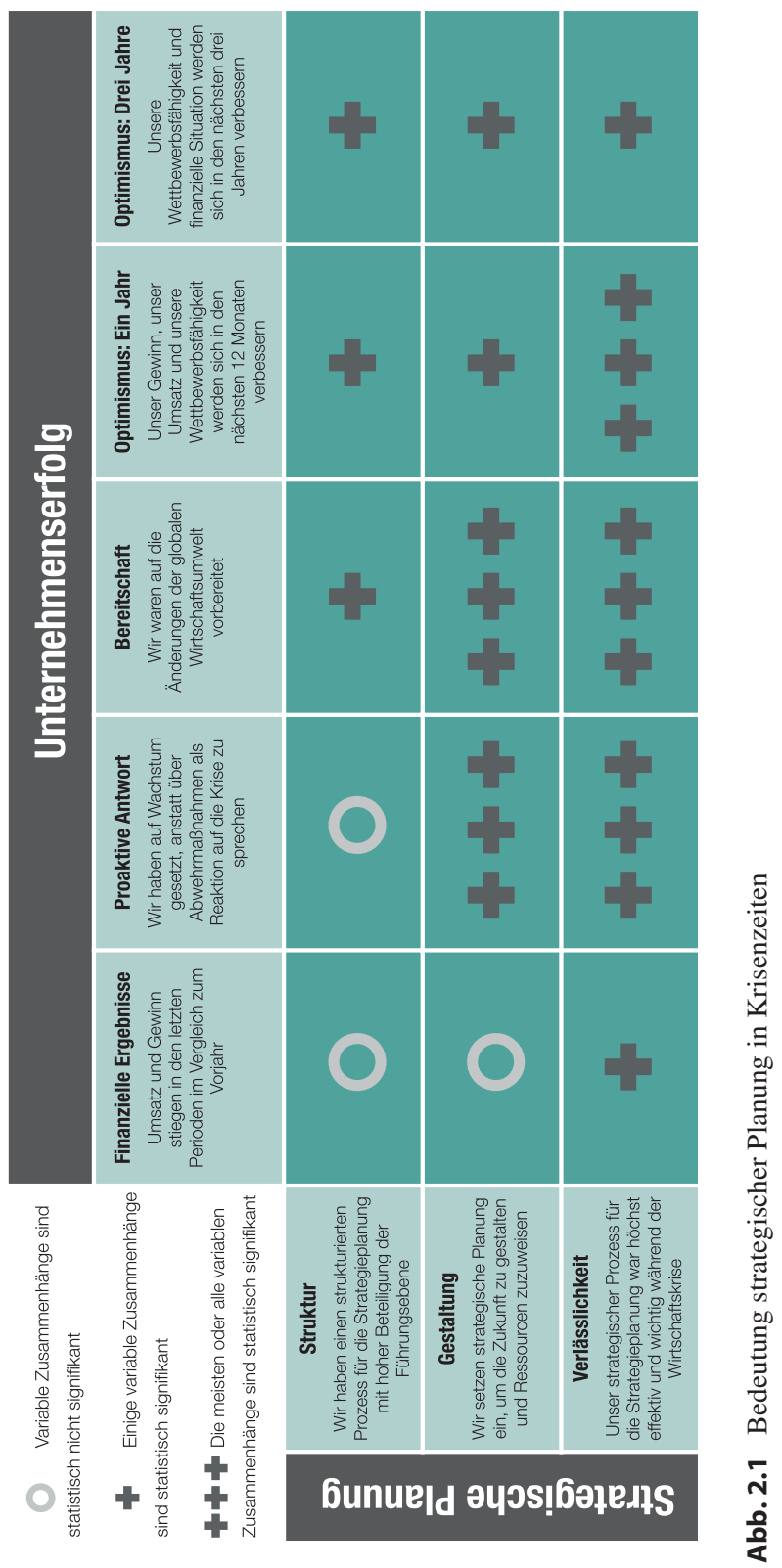




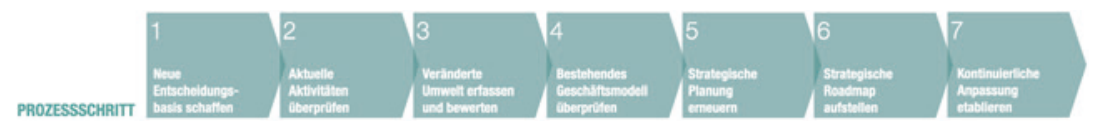

Abb. 2.2 Prozess zur Strategieentwicklung in Krisenzeiten (Übersicht)

auch, dass Unternehmen in der Studie angaben, in zukünftigen Krisen auf Basis ihrer Erfahrungen die strategische Planung und Strategieentwicklung noch weiter zu verstärken. Konkret bedeutet dies zum Beispiel die Durchführung von Trendund Marktanalysen für fundierte Entscheidungen, intensive Einbindung der Entscheidungsträger, sowie Etablierung regelmäßiger Strategieentwicklung und -anpassung [18].

Erkenntnisse aus vergangenen Krisen und den dort verwendeten Erfolgsstrategien von Unternehmen bilden bereits ein gutes Grundgerüst für die Strategieentwicklung in der aktuellen und in zukünftigen Krisen. Da jede Krise jedoch spezifische Besonderheiten mit sich bringt, sind in den nachfolgend beschriebenen Prozess zur Strategieentwicklung in Krisenzeiten auch erste Erkenntnisse aus Sekundärforschung zur aktuellen Coronakrise, sowie aus einer eigenen Ad-hoc-Studie der Autoren vom März 2020 eingeflossen, zu der Unternehmensvertreter aus über 15 unterschiedlichen Branchen sowie Experten aus Wirtschaft, Politik und Wissenschaft befragt wurden. Der hier dargestellte, 7 -stufige Prozess basiert auf der erfolgreichen $5 \mathrm{C}^{\circledR}$-Methodik der Autoren, welche bereits zur strategischen Erneuerung etablierter Unternehmen in mehr als 20 Branchen angewendet, und in diversen Publikationen referenziert wurde [19]. (Abb. 2.2):

1. Neue Entscheidungsbasis schaffen: Kurzfristige und langfristige strategische Ziele und Kriterien anpassen und als Entscheidungsgrundlage nutzen.

2. Aktuelle Aktivitäten überprïfen: Aktuelle Projekte und Maßnahmen anhand der angepassten Ziele und Kriterien überprüfen und je nach Ergebnis anpassen, verstärken, abschwächen oder streichen, sowie Handlungslücken identifizieren.

3. Veränderte Umwelt erfassen und bewerten: Aktuelle und zukünftige gesellschaftliche, wirtschaftliche, politische oder technologische Trends und Einflussfaktoren erfassen und für das eigene Unternehmen als Chance oder Risiko bewerten. 
4. Bestehendes Geschäftsmodell überprüfen: Das bestehende Geschäftsmodell im Kontext der veränderten Rahmenbedingungen durch die Krise bewerten, um ein neues Stärken- und Schwächenprofil des Unternehmens abzuleiten.

5. Strategische Planung erneuern: Kurz- und langfristige strategische Handlungsfelder auf Basis der relevantesten Chancen und Risiken in Bezug auf die externe Umwelt, sowie dominanter Stärken und Schwächen des bestehenden Geschäftsmodells ableiten, und in Bezug auf die in Schritt eins definierten Ziele und Kriterien bewerten und auswählen.

6. Strategische Roadmap aufstellen: Handlungsoptionen innerhalb der ausgewählten strategischen Handlungsfelder definieren und in Bezug auf die Ziele und Kriterien bewerten, um auf dieser Basis eine strategische Roadmap durch die Krise und darüber hinaus abzuleiten.

7. Kontinuierliche Anpassung etablieren: Einen dynamischen Strategieprozesses zur kontinuierlichen Überprüfung und Anpassung der strategischen Roadmap im Zuge weiterer Veränderungen etablieren.

Kernergebnis des hier skizzierten Prozesses ist eine strategische Roadmap mit kurz-, mittel- und langfristigen Projekten und Maßnahmen für die Zeit während und nach der Krise, um die vom Unternehmen gewählten Ziele bestmöglich $\mathrm{zu}$ erreichen. Auf diese Weise wird zum einen eine gemeinsame, klare Vision für alle betroffenen internen und externen Stakeholdern in Zeiten von Volatilität und Ungewissheit geschaffen. Zum anderen entsteht eine optimale Basis für die gezielte, geplante und geordnete Umsetzung der Maßnahmen, und somit für kurz- und langfristige Stabilität und Wachstum des Unternehmens. Die durch den Prozess erarbeitete strategische Roadmap gilt es dabei in Form eines dynamischen Strategieprozesses kontinuierlich zu überprüfen und anzupassen.

\subsection{Neue Entscheidungsbasis schaffen: Strategische Ziele und Kriterien}

„1929 war ein negativer Nachfrageschock durch einen Börsencrash und dann mehrere Wellen von Bankenpleiten. [In der Coronakrise] haben wir einen negativen Angebotsschock - Fabriken werden geschlossen, weil es zu unsicher ist, zur Arbeit zu gehen, die Lieferketten wurden unterbrochen. Hinzu kommt ein negativer Nachfrageschock, da die Menschen ihre Gehaltsschecks verlieren und die Verbraucher aus Angst aufhören zu konsumieren. Zentralbanken und Finanzministerien können zwar einen Anreiz zur Unterstützung der Nachfrage bieten. Aber sie können nichts tun, um das Problem auf der Angebotsseite zu lösen [20].“

Prof. Barry Eichengreen, UC Berkeley, März 2020 
In unvorhergesehenen Krisensituationen wie der Coronakrise stehen Unternehmen plötzlich vor gänzlich neuen Rahmenbedingungen. Da diese in bisherigen strategischen Planungen nicht berücksichtigt wurden, oder vielmehr nicht berücksichtig werden konnten, entfällt die Validität vieler Ziele und Kriterien, die vor der Krise definiert und beschlossen wurden.

Diese drastischen und plötzlichen Veränderungen bzw. Schocks lassen sich in die Kategorien Nachfrage-, Angebots-, und Finanzschock einteilen. Der Internationale Währungsfonds erklärt diese am Beispiel der Coronakrise wie folgt:

- Nachfrageschock: Zur Eindämmung des Virus sind Menschen weltweit gezwungen, zu Hause zu bleiben bzw. ihre Kontakte einzuschränken. Dies führt dazu, dass bestimmte Angebote für einen längeren Zeitraum nicht mehr oder nur noch eingeschränkt wahrgenommen werden können. Dies schadet ganzen Branchen wie zum Beispiel dem Einzelhandel, der Gastronomie oder der Reisebranche. In Erwartung einer möglichen Wirtschaftskrise halten sich die Konsumenten auch insgesamt mit Käufen zurück, sodass selbst Onlinekanäle nur verhältnismäßig schwach profitieren können.

- Angebotsschock: Durch Fabrikschließungen in diversen Ländern, angefangen mit China, geraten globale Lieferketten in schwere Turbulenzen. Betriebe müssen sich teilweise über Nacht auf die Suche nach neuen Lieferanten begeben. Frachtschiffe bleiben mangels Fracht in Häfen liegen, während der Frachtraum im Flugverkehr teurer wird, da Bedarfe schnell gedeckt werden müssen.

- Finanzschock: Der gleichzeitige Angebots- und Nachfrage-Schock, das Chaos in den Lieferketten, sowie die Erosion von Vertrauen und Zuversicht bei Konsumenten und Produzenten bringt schließlich den Finanzsektor in die Krise. Dieser hat auf der einen Seite Kreditausfälle und Liquiditätsengpässe bei den Unternehmen zu verkraften, während auf der anderen Seite die Nachfrage nach profitablen Anlageprodukten sinkt [21].

In Anbetracht solch gravierender Herausforderungen besteht für Unternehmen die Notwendigkeit, bisherige strategische Ziele und Kriterien zu hinterfragen und gegebenenfalls an die neue Situation anzupassen. In einer akuten Krisensituation ist dabei insbesondere auf eine Unterscheidung zwischen kurz- und langfristigen Zielen zu achten, da diese erwartungsgemäß stark differieren können. In der von den Autoren durchgeführten Ad-hoc-Studie zur Coronakrise dominierten bei den befragten Unternehmen beispielsweise kurzfristig Ziele in Bezug auf die eigene finanzielle Sicherheit, während langfristig Wachstumsziele wieder stärker im Fokus standen. 
Um die im Zuge der Krise angepassten Ziele und Kriterien für die konkrete Entscheidungsfindung für bzw. gegen Maßnahmen während und nach der Krise nutzbar zu machen, bietet es sich an, diese in eine individuelle Entscheidungsmatrix zu überführen.

\section{Instrument 1: Entscheidungsmatrix auf Basis strategischer Ziele und Kriterien}

Die Basis für effizientes, fokussiertes Handeln in der Krise (und darüber hinaus) bilden eindeutige und messbare strategische Ziele und Kriterien. Auf dieser Grundlage können mögliche Handlungsoptionen zielgerichtet entwickelt, sowie transparent bewertet und ausgewählt werden. Das Vorgehen gliedert sich dabei in drei Schritte:

\section{Definition von Zielen und Kriterien}

Die Definition messbarer Ziele und Kriterien bildet die Grundlage für die Strategieentwicklung. Im Kontext einer Krise gilt es zunächst, bestehende kurz- und langfristige Ziele und Kriterien zu hinterfragen und entsprechend der veränderten Rahmenbedingungen für das Unternehmen anzupassen, zu streichen oder zu ergänzen. Im ersten Schritt wird dazu eine Longlist aus bestehenden sowie potenziell relevanten, neuen Zielen und Kriterien erstellt (Abb. 2.3).

2. Ranking und Auswahl von Zielen und Kriterien

Selbst in einer Krise liegen die strategischen kurz- und langfristigen Ziele nicht für alle Unternehmen gleichermaßen auf der Hand. Und unterschiedliche Entscheidungsträger haben möglicherweise unterschiedliche Sichtweisen in Bezug auf die externen Veränderungen und die Bedeutung einzelner Ziele und Kriterien. Um diesen Herausforderungen Rechnung zu tragen, gilt es, die zuvor definierte Auswahl an möglichen Zielen und Kriterien aus der Longlist von den relevanten Entscheidungsträgern im Unternehmen individuell bewerten zu lassen (z. B. per Online-Fragebogen). Zur Bewertung der Ziele und Kriterien bietet sich eine Priorisierung in Bezug auf die jeweilige kurzund langfristige Relevanz $(1=$ niedrig, $3=$ hoch) an (Abb. 2.3). Die Auswertung der individuellen Bewertungsergebnisse dient anschließend als Basis für eine gemeinsame, finale Auswahl. Bestenfalls findet diese im Rahmen eines persönlichen oder virtuellen Workshops der Entscheidungsträger statt. Dieser Prozess dient neben der Fokussierung auf eine sinnvolle Auswahl von Zielen und Kriterien auch dazu, unterschiedliche Einschätzungen von Entscheidungsträgern in ein gemeinsames Zielbild zu überführen. 


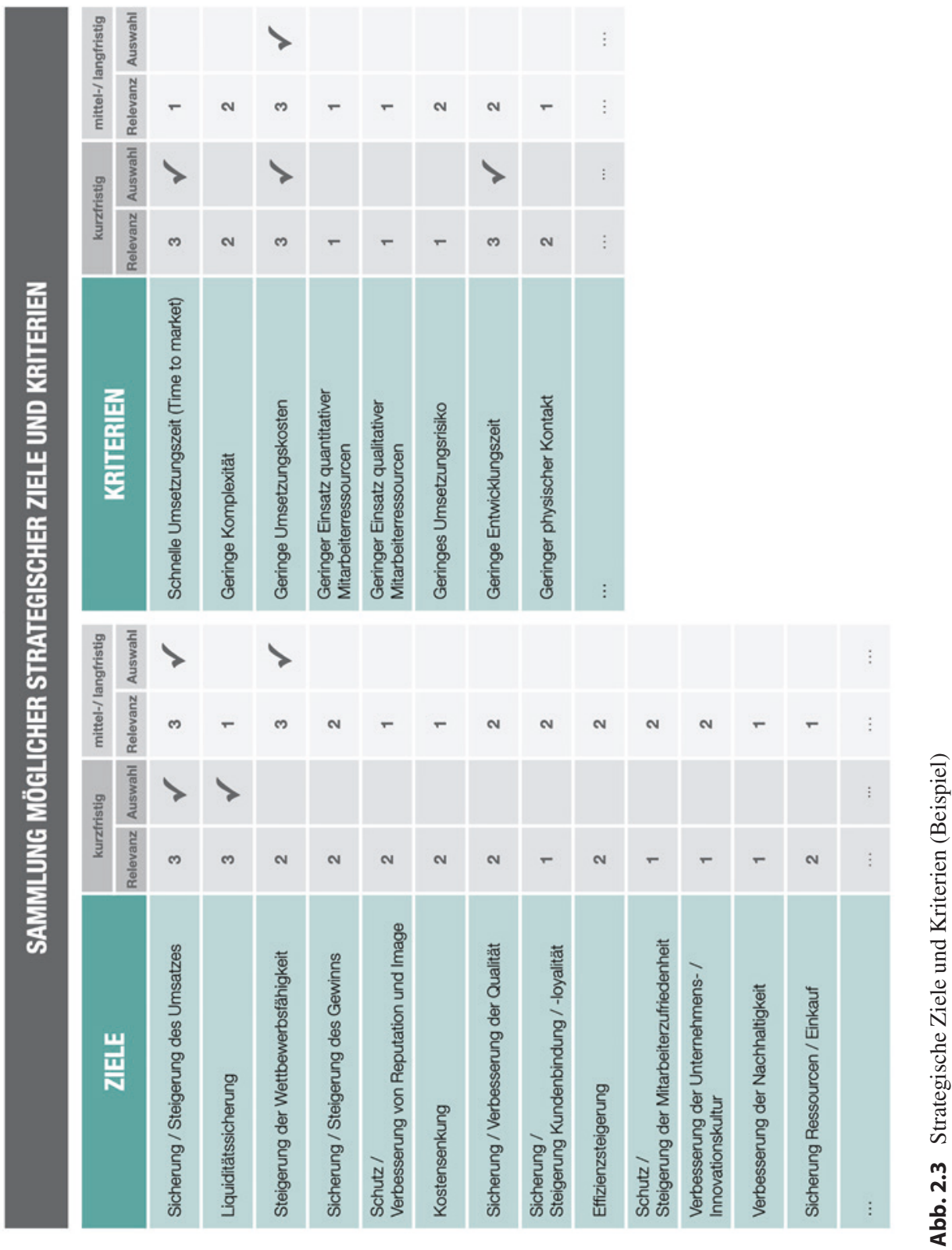




\section{Entscheidungsmatrix auf Basis ausgewählter Ziele und Kriterien}

Um die ausgewählten Ziele und Kriterien für die Bewertung von Handlungsfeldern und Maßnahmen operativ nutzbar zu machen, gilt es, diese im nächsten Schritt in eine Matrixansicht zu überführen. Die sogenannte Entscheidungsmatrix kombiniert die wichtigsten strategischen Ziele (Y-Achse) mit der Umsetzungszeit (X-Achse). Durch die Verortung von möglichen strategischen Handlungsfeldern oder konkreten Maßnahmen innerhalb der Entscheidungsmatrix, lassen sich für diese transparent und schnell jeweils passende Handlungsoptionen ableiten. So sind in Krisenzeiten insbesondere „Quickwins“ gefragt, während ausgewählte „Long Term Wins“ für Überleben und Wachstum nach der Krise sorgen. Da sich auf der Y-Achse kurz- und langfristige Ziele gleichermaßen wiederfinden, bietet es sich an, bei Handlungsfeldern und Maßnahmen die kurzfristig umgesetzt werden können, eine Bewertung nach den kurzfristigen Zielen vorzunehmen, und im Falle einer längerfristigen Umsetzung in Bezug auf die Erreichung der langfristigen Ziele zu bewerten. Falls bei der Auswahl auch bestimmte Kriterien berïcksichtigt werden sollen, kann deren Ausprägung beispielsweise durch die Umrandung einer bestimmten Handlungsoption, oder alternativ durch unterschiedliche Größen verdeutlicht werden (Abb. 2.4).

\subsection{Aktuelle Aktivitäten überprüfen: Zielgerichtete Portfolio-Optimierung}

„Ein Schlüssel zur Bewältigung der Coronakrise ist die sehr kurzfristige Vergabe von Krediten in Milliardenhöhe. Als staatliche Förderbank bedeutet dies unter enormen Druck in 2 bis 3 Tagen Dinge umzusetzen, die unter normalen Umständen Monate dauern. In derartigen Notsituationen verwandelt sich banktypische Risikoaversion plötzlich in Pragmatismus. Es gilt schnelle Entscheidungen zu treffen, die richtigen Maßnahmen auszuwählen und diese fokussiert anzugehen.“

Michael Strauß, CDO KfW, April 2020

Gemeinsam mit den Zielen verändern sich in Krisenzeiten auch plötzlich die Prioritäten. Was gestern richtig war, kann heute falsch und morgen fatal sein. Umso wichtiger ist es, schnell und objektiv bestehende Aktivitäten und Projekte neu zu bewerten und gegebenenfalls anzupassen, zu verstärken, abzuschwächen, oder zu streichen. So haben Unternehmen zum Beispiel im Zuge der Coronakrise teilweise innerhalb von wenigen Tagen und Wochen ihre Aktivitäten an die Erfordernisse der neuen Situation angepasst: TV-Sender verstärkten 

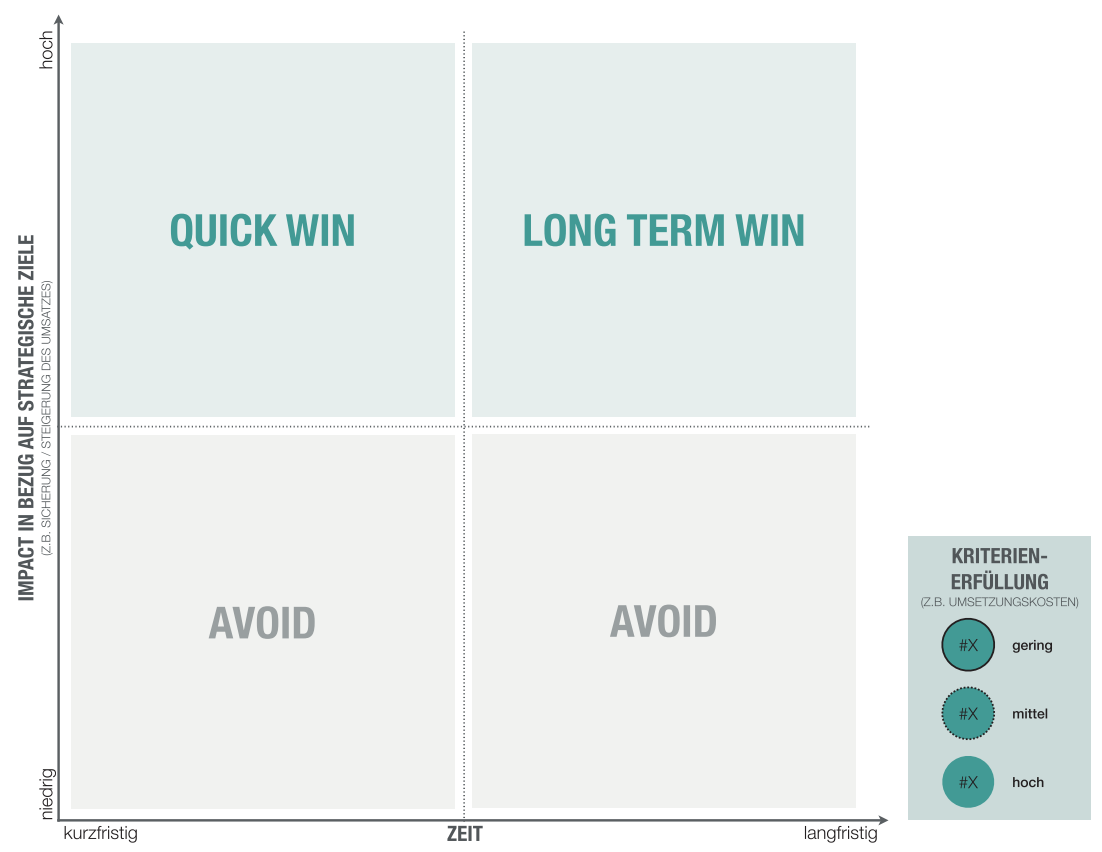

Abb. 2.4 Entscheidungsmatrix

aufgrund von Schulschließungen ihre Wissensprogramme für Kinder, der PayTV-Sender Sky erstattete den geschlossenen Restaurants und Bars die Kosten des Abonnements, McDonalds verlieh seine Mitarbeiter an Aldi, der französische Luxuskonzern LVMH stellte Teile seiner Produktion von Parfüm auf Desinfektionsmittel um, und die Lufthansa entschied, mit einigen der stillgelegten Boeing-747-Passagiermaschinen nun reine Frachtflüge für stark nachgefragte Medizin- und Schutzausrüstung durchzuführen.

Wichtig bei all diesen kurzfristigen Entscheidungen ist es, blinden Aktionismus zu vermeiden. Vielmehr sollte zielgerichtet und effizient vorgegangen werden. Dabei müssen neben kurzfristigen Erfordernissen während der Krise ebenso die langfristigen Herausforderungen über die Krise hinaus berücksichtigt werden. Dies impliziert unter Umständen auch, bei der Entscheidungsfindung zwischen kurzfristigem Nutzen und langfristigem Schaden für das Unternehmen abzuwägen. 
Dies kann beispielsweise bei der Entlassung von Mitarbeitern in einer akuten Krisensituation zutreffen. Zunächst wirkt eine solche Maßnahme sinnvoll, da auf diese Weise kurzfristig Kosten eingespart und somit die Liquidität des Unternehmens gesichert werden kann. Dies war auch der Leitgedanke von United Airlines und US Airways in der 9/11 Krise. Beide Fluggesellschaften entließen hunderte Piloten und Mitarbeiter. Nachträglich, im Kontext einer sich erholenden Wirtschaft, stellte sich diese Strategie als äußerst problematisch heraus. Der Börsenkurs von United Airlines hatte sich selbst vier Jahre nach der Krise gerade einmal um $12 \%$ erholt, der von US Airways um nur $20 \%$. Ganz anders das Bild bei Southwest Airlines. Das Unternehmen hielt während der 9/11 Krise an allen Mitarbeitern fest und konnte 2002 bereits weitere Personen einstellen. Vier Jahre nach der Krise hatte sich der Börsenkurs der Fluggesellschaft bereits um $92 \%$ erholt. Auch wenn dies auf den ersten Blick anekdotisch erscheint, wurde mittlerweile wissenschaftlich erwiesen, dass die Erholung der Aktienkurse von US-Fluggesellschaften im Vergleich zu ihrem Vorkrisenniveau signifikant und negativ mit dem Ausmaß der Entlassungen zur Zeit der Krise zusammenhängt. Dies ist durch multiple Faktoren zu erklären, welche die Organisation bei Entlassungen schwächen. Dazu zählen zum Beispiel die Zerstörung der zwischenmenschlichen Beziehungen und Unternehmenskultur, erhöhter Widerstand gegen Veränderungen und Konservatismus, aufkommende Konflikte, sowie erhöhte Selbstsucht und freiwillige Fluktuation mit entsprechender Verschlechterung der Teamarbeit und Zusammenarbeit [22].

\section{Instrument 2: Portfoliobewertung und -steuerung}

Die strategischen Ziele und Kriterien wurden bereits auf die aktuelle Situation angepasst und eine Entscheidungsmatrix zur schnellen und transparenten Bewertung geschaffen (siehe Instrument 1). Auf dieser Basis kann nun schnell und fundiert bewertet werden, welche Aktivitäten im Kontext der Krisensituation auf die Unternehmensziele einzahlen und welche nicht. Und folglich auch, inwieweit strategische Projekte fortgeführt, verstärkt, pausiert, gestoppt oder angepasst werden müssen. Auch eventuelle Handlungslücken werden im Zuge dieser Analyse identifiziert. Das konkrete Vorgehen zur Portfoliobewertung- und steuerung gliedert sich in drei Schritte:

\section{Konsolidierung strategischer Projekte und Aktivitäten}

Im ersten Schritt gilt es, sämtliche kurz-, mittel- und langfristige, strategisch relevante Projekte und Maßnahmen zu sammeln (Abb. 2.5). Darunter fallen sowohl laufende Aktivitäten und Projekte als auch neue Projektideen. Sollten die Projekte bislang noch nicht an geeigneter Stelle im Unternehmen 

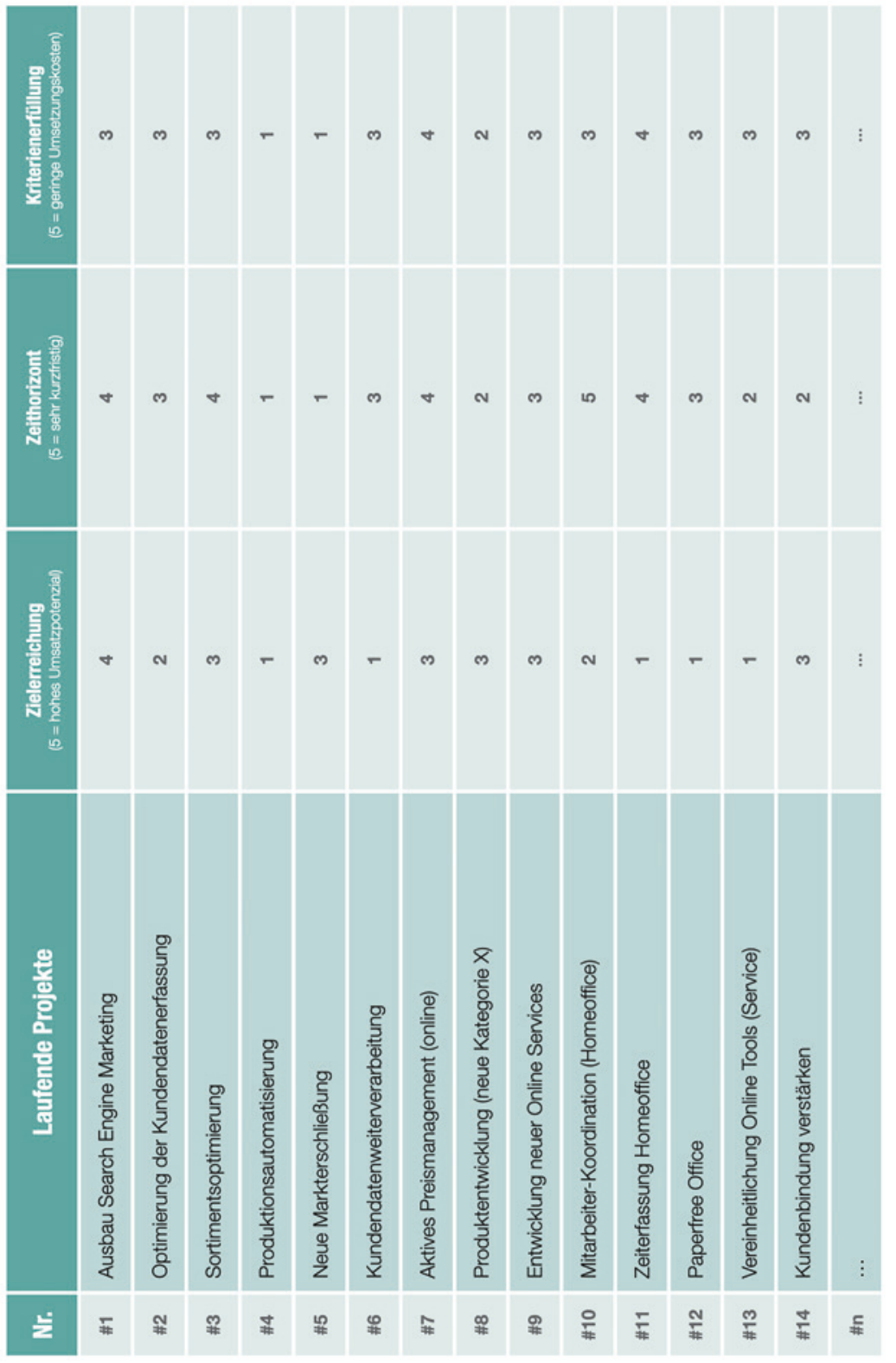

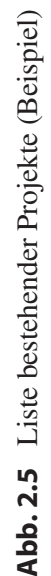


dokumentiert sein, ist eine umso engere Zusammenarbeit der involvierten Abteilungen gefragt. Idealerweise sollte sich dabei eine Einheit, wie beispielsweise die Strategieabteilung, das Portfoliomanagement oder Business Development, verantwortlich zeichnen und die Sammlung relevanter Projekte von Abteilungen wie Marketing, Finanzen, Personal oder Vertrieb koordinieren.

\section{Bewertung und Verortung strategischer Projekte}

Ist eine Übersicht sämtlicher relevanter Projekte und Maßnahmen erstellt, können diese zunächst in Bezug auf ihren (potenziellen) Beitrag auf die zuvor definierten Ziele und Kriterien bewertet werden. Anschließend werden die einzelnen Aktivitäten zur visuellen Darstellung in der Entscheidungsmatrix verortet (Abb. 2.6). Auf der Y-Achse wird der jeweilige Impact in Bezug auf die strategischen Ziele abgetragen, auf der X-Achse der mögliche Umsetzungszeitpunkt. Da die Umsetzungsdauer einer Aktivität oftmals mit deren Aufwand bzw. Komplexität korreliert, kann die Position auf der X-Achse in der Regel auch Aufschluss über Dauer, Aufwand und Komplexität geben. Über die visuelle Unterscheidung der Umrandung einzelner Projekte oder Maßnahmen in der Matrix kann darüber hinaus eine Aussage über relevante Kriterien, wie zum Beispiel die Umsetzungskosten, abgebildet werden. Die Verortung der Aktivitäten kann dabei sowohl digital als auch ana$\log$, zum Beispiel auf einer großen Wand mit Post-Its, erfolgen. Unabhängig davon, wie die Materialisierung der Matrix konkret erfolgt, das Ergebnis ist stets wertvoll: Eine transparente Übersicht über sämtliche potenzielle kurz-, mittel- und langfristige Aktivitäten für die Zeit während und nach der Krise. Und dies auf Basis eines gemeinschaftlichen, im Entscheiderkreis verabschiedeten Zielbildes.

3. Analyse und Anpassung des Portfolios inkl. Handlungslücken

Die mit (potenziellen) Aktivitäten gefüllte Entscheidungsmatrix bildet nun die Grundlage für erste strategische Entscheidungen. Projekte, die sich in den unteren beiden Quadranten der Matrix befinden, sollten bestenfalls kurzfristig gestoppt oder zumindest pausiert werden, um zusätzliche Kapazitäten freizusetzen. Dabei ist jedoch bei jedem einzelnen Projekt bzw. jeder Maßnahme zu prüfen, ob es sich dabei um eine systemkritische Aktivität handelt, die zwingend fortgesetzt werden muss, beispielsweise ein wichtiges Infrastrukturprojekt. Projekte, die sich in den beiden oberen Quadranten der Matrix befinden, sollten prinzipiell fortgeführt und gegebenenfalls sogar verstärkt werden. Ist das jeweilige Projekt bzw. die Maßnahme noch nicht in der Umsetzung, kann an dieser Stelle eine positive Entscheidung zu deren Umsetzung fallen. Dabei sind „Quick Wins“, also Aktivitäten die bereits 


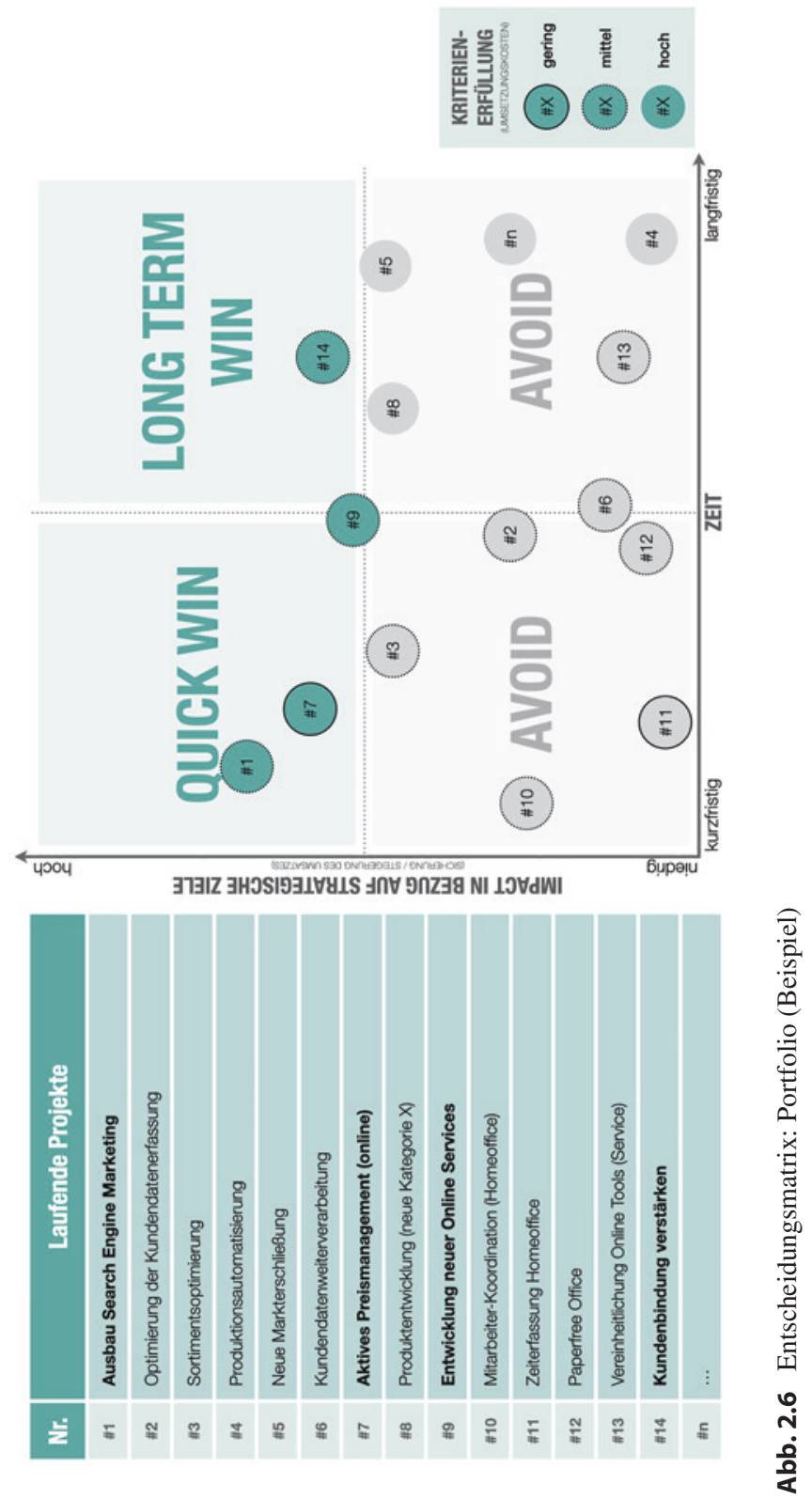


kurzfristig - und bestenfalls mit weniger Aufwand- durchgeführt werden können, in Krisenzeiten von besonders hoher Relevanz. Wie in den vorherigen Kapiteln bereits erläutert wurde, sollten jedoch auch "Long Term Wins“ nicht vernachlässigt werden, um langfristige Chancen im Kontext der neuen Rahmenbedingungen zu nutzen. Falls die Entscheidungsmatrix keine oder wenige (potenzielle) Aktivitäten in den oberen beiden Quadranten zeigt, ist der Handlungsbedarf zur Erarbeitung weiterer, neuer Projekte und Maßnahmen für die Zeit während und nach der Krise besonders groß. Doch auch eine mit vielen Projekten und Maßnahmen gefüllte obere Matrix-Hälfte kann trügerisch sein. Schließlich wurden bis zu diesem Punkt im Prozess weder neue Chancen und Risiken, die sich im Zuge der Krise ergeben, noch veränderte Stärken und Schwächen in Bezug auf das eigene Geschäftsmodell, aufgedeckt. Folglich sollte die Entscheidungsmatrix eher als Startpunkt für die weitere Strategiearbeit betrachtet werden.

\title{
2.3 Veränderte Umwelt erfassen und bewerten: Chancen und Risiken
}

\begin{abstract}
„Der [Corona-]Ausbruch hat nicht nur die Finanzmärkte und das kurzfristige Wachstum unter Druck gesetzt: Er hat eine Neubewertung vieler Annahmen über die Weltwirtschaft ausgelöst, wie zum Beispiel unsere Begeisterung für Just-in-Time-Lieferketten, oder unsere Abhängigkeit von internationalen Flugreisen. Noch tiefgreifender ist, dass die Menschen weltweit die Art und Weise, wie wir arbeiten, einkaufen, reisen und uns versammeln, grundlegend überdenken. Wenn wir diese Krise überwinden, wird die Welt anders aussehen. Die Psychologie der Investoren wird sich ändern. Das Geschäft wird sich ändern. Der Konsum wird sich ändern. Und wir werden stärker auf unsere Familien und aufeinander angewiesen sein, um sicher zu sein [23].“
\end{abstract}

Larry Fink, CEO Blackrock, März 2020

Die Worte des Blackrock-CEOs Larry Fink aus seinem Investorenbrief zur Coronakrise im Frühjahr 2020 beschreiben das enorme Veränderungspotenzial von Krisen. Tatsächlich können größere Schocks zu grundlegenden Veränderungen in Verhaltensweisen, Bedürfnissen, Werten und Normen führen, die wiederum den Weg für neue Politik, Arbeitsweisen und Konsumverhalten ebnen. Dies zeigt ein Blick auf vergangene „Tiefenkrisen“. So wird die Pest, welche im 14. Jahrhundert 25 bis 30 Mio. Menschenleben kostete, von Historikern als Ursprung der Renaissance gesehen. Aufgrund knapper Arbeitsressourcen nach der Krise wurden Feudalismus und Leibeigenschaft beendet. Damit wurde nicht nur Raum für das Erblühen der Wirtschaft, sondern auch für vollkommen neue Ideen geschaffen. 
Der zweite Weltkrieg war nicht nur Ausgangspunkt eines neuen Multilateralismus, sondern ebnete auch den Weg für Frauen als Arbeitskräfte, da diese oftmals die Tätigkeiten ihrer Männer im Krieg weiterführen mussten. Aber auch weniger tief greifende Krisen haben kurz- und langfristige Veränderungen gebracht. So hat sich zwar nach ,9/11“ das Reiseaufkommen schnell wieder erholt, doch noch heute leben wir mit zahlreichen neuen Sicherheitsregularien im Flugverkehr. Die Finanzkrise wird wiederum von vielen als Anfang vom Ende des Neoliberalismus gesehen, da die zuvor propagierte, unsichtbare Hand des Marktes de facto nicht mehr für dessen Regulierung ausreichte, und so die Rolle des Staates wieder wichtiger wurde [24].

Die Coronakrise hat bereits kurzfristig schwerwiegende Veränderungen in der gesamten Gesellschaft verursacht, unter anderem in Bezug auf die Bedeutung virtueller Arbeitsweisen und die Digitalisierung im Allgemeinen. Damit ist sie am ehesten mit der SARS-Krise 2002/2003 in Asien vergleichbar, welche dort als Geburtsstunde des E-Commerce von Alibaba \& Co gilt [7]. Eine Umfrage von Morning Consult im Kontext der Coronakrise zeigt, dass viele US-Bürger ihr Einkaufsverhalten kurzfristig zugunsten von Online-Kanälen verändert haben. Auch in Deutschland erhöhte sich die Zahl der Suchanfragen nach Online-Lebensmittelhändlern wie Amazon Fresh zu Beginn der Coronakrise um das zehnfache [25]. Eine solche Verschiebung zum Online-Einkauf könnte durchaus auch langfristig ein verändertes Kaufverhalten mit sich bringen. Langjährige E-Commerce-Verweigerer, wie beispielsweise viele ältere Menschen, sind in der Krisensituation unter Umständen auf die Onlinekanäle angewiesen und könnten diese auch nach der Pandemie weiter nutzen [26]. Gleiches gilt für die Digitalisierung in Unternehmen, da Menschen plötzlich gezwungen sind, im Home-Office zu arbeiten, und dabei möglicherweise feststellen, dass dies trotz aller Vorbehalte funktioniert. Ein Effekt, der auch langfristig anhalten kann, da im Zuge der akuten Krise die notwendige Infrastruktur bereitgestellt werden musste, die in der Regel auch nach der Krise weiter zur Verfügung steht Viele Unternehmen haben im Zuge der aktuellen Krise zudem erkannt, dass sie für globale Krisen nicht ausreichend gerüstet sind und stellen teilweise bereits ihr Verhalten um, wie Zukunftsforscher Matthias Horx beschreibt [6].

„Die globale Just-in-Time-Produktion mit riesigen verzweigten Wertschöpfungsketten, bei denen Millionen Einzelteile über den Planeten gekarrt werden, hat sich überlebt. Sie wird gerade demontiert und neu konfiguriert. Überall in den Produktionen und Service-Einrichtungen wachsen wieder Zwischenlager, Depots, Reserven. Ortsnahe Produktionen boomen, Netzwerke werden lokalisiert, das Handwerk erlebt eine Renaissance. Das Global-System driftet in Richtung GloKALisierung: Lokalisierung des Globalen“.

Matthias Horx, Zukunftsforscher, März 2020 
Die aus der Coronapandemie resultierenden politischen Einschnitte in das öffentliche Leben zwingen Menschen dazu, auf viele nicht-lebensnotwendige Freizeitaktivitäten und Dienstleistungen wie Kunst, Kultur, Reisen, oder Konferenzen zu verzichten. Wird dies die Bedeutung derartiger Aktivitäten in Zukunft steigern, weil diese nicht mehr als selbstverständlich wahrgenommen werden? Oder werden Menschen feststellen, dass sie vielleicht gar nicht an jedem Event teilnehmen, jeden neuen Kinofilm sehen und nicht regelmäßig zum Schönheitssalon gehen müssen? Die langfristigen Veränderungen, die ihren Ursprung in der Krise finden, lassen sich auch als „Hysterese-Effekt“ bezeichnen. In der Physik beschreibt der Hysterese-Effekt einen temporären Impuls, der eine langfristige Wirkung auslöst, wie es der Managementforscher Hermann Simon an einem Beispiel beschreibt [27].

„,Die folgende E-Mail, die mir ein normalerweise viel reisender Bekannter am 13. März schrieb, deutet auf einen Hysterese-Effekt hin: „Ich kenne Dutzende von Leuten, die noch nie Lebensmittel online eingekauft haben und die das jetzt tun. Ich gehöre dazu. Ich werde nicht mehr auf offline Lebensmitteleinkauf wechseln, nachdem ich mich jetzt einmal vertraut gemacht habe mit den online Lebensmittelangeboten."

\section{Hermann Simon, Wirtschaftsprofessor, Gründer, Simon-Kucher \& Partners.} März 2020

Wie in jeder Krise werden sich auch im Kontext der Coronakrise Unternehmen also darauf einstellen müssen, dass sich die Realität nachhaltig verändern wird. Auch wenn noch nicht final absehbar ist, welche konkreten Veränderung Bestand haben werden, lohnt es sich, kurzfristige Veränderungen bereits frühzeitig im Unternehmenskontext zu bewerten, um daraus mögliche Implikationen für langfristige Chancen und Risiken abzuleiten. Hierbei kann es helfen, unterschiedlichen Szenarien zu entwerfen. Dabei ist es nicht entscheidend, dass eines der Szenarien tatsächlich eintritt, sondern dass der Raum für Möglichkeiten bewusst geöffnet wird, wie Professor Müller-Stewens von der Universität St. Gallen beschreibt: „Aus der Hirnforschung wissen wir, dass Menschen, die Szenarien entwickelt haben, schneller als andere erkennen, was auf sie zukommt [28].“

Unternehmen hilft es an dieser Stelle, auf Wissen und Forschung von Experten zurückzugreifen. So hat zum Beispiel das Zukunftsinstut verschiedene Szenarien für die Entwicklung ihrer Megatrends im Kontext der Coronakrise erstellt. Dabei zeigt sich, dass fünf dieser Megatrends für sämtliche Szenarien von zentraler Bedeutung sind, auch wenn deren genaue Richtung noch nicht bekannt ist. Dabei handelt es sich um die Megatrends Sicherheit, Gesundheit, Konnektivität, Individualisierung und Globalisierung. Abhängig von deren Entwicklung konnten 
vier Szenarien identifiziert werden, die nun Möglichkeiten für die Zukunft beschreiben (Abb. 2.7) [29].

Eine ganzheitliche Szenarienplanung wäre Inhalt genug für ein weiteres Buch und ist auch nicht für jedes Unternehmen gangbar, da dazu weitreichende Expertise erforderlich ist. Es lohnt sich jedoch, zumindest eine generelle Entscheidungsbasis zu schaffen, indem relevante externe Einflussfaktoren strukturiert gesammelt und in Bezug auf Chancen und Risiken für das Unternehmen bewertet werden. Aus dieser Analyse lassen sich anschließend Handlungsbedarfe und Möglichkeiten für die Zeit während und nach der Krise ableiten. Dabei gilt es zu berïcksichtigen, dass sich Einflussfaktoren auch innerhalb des Krisenzeitraums mitunter stark verändern können. Aus diesem Grund ist anzuraten, deren Entwicklung kontinuierlich zu beobachten.

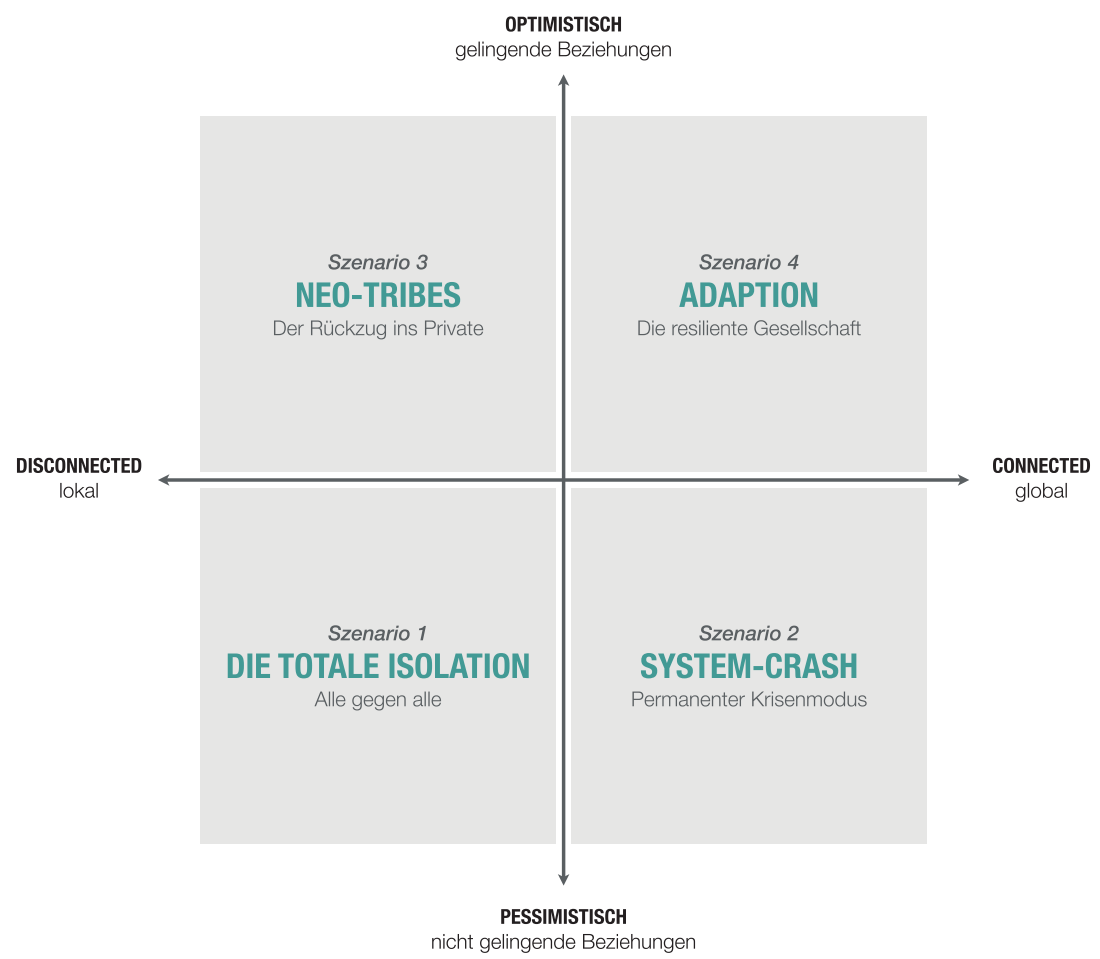

Abb. 2.7 Szenarien des Zukunftsinstituts 


\section{Instrument 3: Umweltanalyse}

Um eine auf das eigene Unternehmen bezogene Erfassung und Bewertung der veränderten externen Einflussfaktoren vorzunehmen, bietet sich eine strukturierte Umweltanalyse an, welche aktuelle und zukünftige Entwicklungen in Politik, Wirtschaft, Gesellschaft und Technologie beinhaltet und sich in drei Schritte gliedert.

\section{Analyse externer Einflussfaktoren}

Um eine systematische Erfassung der Einflussfaktoren zu erreichen, wird die Umwelt zunächst in thematische Suchfelder unterteilt. Hier bieten sich Bereiche wie Markt, Technologie, Gesellschaft, Politik und Ökologie an. Innerhalb dieser Felder werden anschließend relevante Einflussfaktoren erfasst. Einen guten Startpunkt zu deren Identifikation bilden bestehende Analysen wie zum Beispiel die Megatrend-Map des Zukunftsinstituts, oder spezifische Trendreports (Abb. 2.8).

Bezüglich der Einflussfaktoren empfiehlt es sich, jeweils zu prüfen, ob diese im Unternehmenskontext eher positiv oder negativ zu bewerten sind, und inwieweit sie potenziell eher kurz- oder langfristig zum Tragen kommen. Nicht jeder Einflussfaktor ist dabei für das eigene Unternehmen von ähnlich hoher Relevanz. Es gilt: Je relevanter ein einzelner Einflussfaktor ist, desto größer ist die damit verbundene potenzielle Chance bzw. das potenzielle Risiko, dass von diesem Faktor für das Unternehmen ausgeht (Abb. 2.9).

2. Bewertung und Verortung externer Einflussfaktoren

Im nächsten Schritt gilt es, die identifizierten Einflussfaktoren entsprechend ihrer Bewertung als Chance oder Risiko, sowie ihrer zeitlichen Relevanz und Bedeutung für das Unternehmen in die passende Auswahlmatrix zu übertragen (Abb. 2.10). Für verschiedene Szenarien können auch mehrere Matrizen gebildet werden, in denen die diversen Einflussfaktoren mit unterschiedlichen Bewertungen abgetragen werden. Die Beobachtung der Einflussfaktoren über den Zeitverlauf erlaubt dann die kontinuierliche Bewertung und Anpassung der Szenarien. Diese Szenariobetrachtung kann auch in den weiteren Schritten des hier beschriebenen Strategieprozesses angewandt werden. Aus Gründen der Vereinfachung wird im weiteren Verlauf allerdings nur jeweils ein Szenario betrachtet.

3. Auswahl von Chancen und Risiken zur Strategieentwicklung

Auf Basis der zuvor erstellten Matrix können nun die relevantesten kurzund langfristigen Chancen und Risiken ausgewählt werden, um daraus neue potenzielle Handlungsfelder für das Unternehmen abzuleiten (Abb. 2.11). Die Auswahl erfolgt dabei entsprechend der höchsten Bedeutung einer Chance bzw. eines Risikos für das Unternehmen, sowie ggf. ergänzender Diskussionen mit Entscheidungsträgern und Experten. 


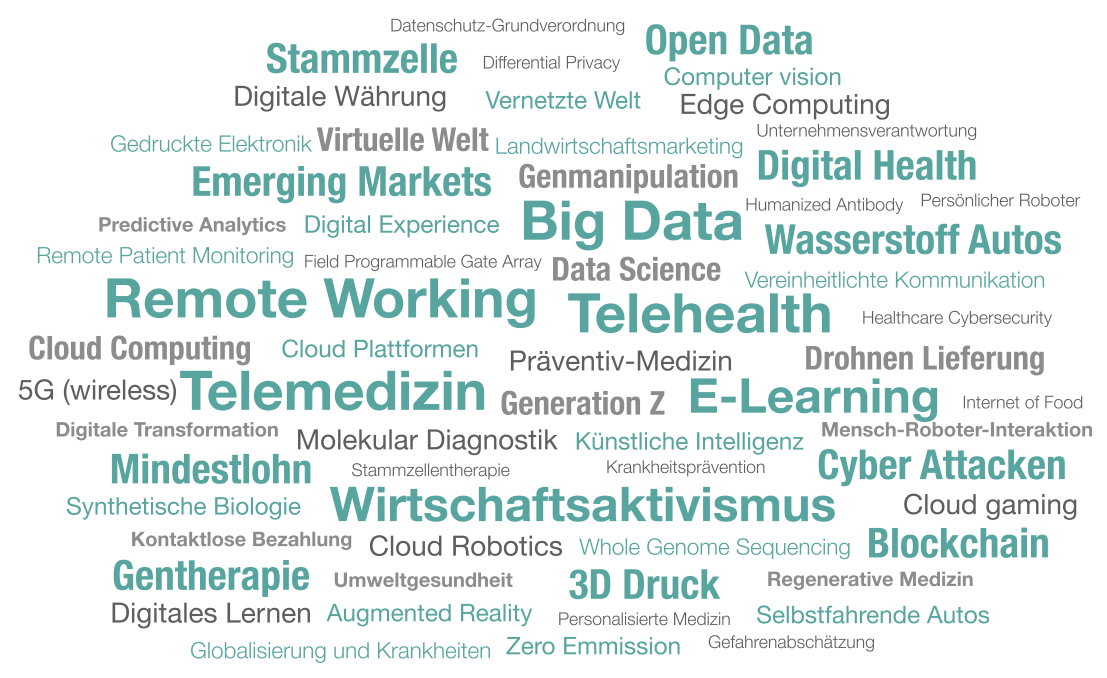

Abb. 2.8 Ausgewählte Trends in der Coronakrise

\title{
2.4 Bestehendes Geschäftsmodell überprüfen: Stärken und Schwächen
}

\begin{abstract}
„Geschäftsmodelle müssen grundsätzlich überdacht werden. Was betriebskritisch ist, muss wieder ingesourced werden - Stichworte Wertschöpfungstiefe, Antifragilität und dynamikrobuste Systeme. Das ist das Gegenkonzept zum Effizienzdenken der Vergangenheit. Es geht darum Redundanzen aufzubauen, um sich gegen Krisen abzusichern. Dabei ist jetzt auch die Zeit für Innovation. Jetzt ist die Zeit die Dinge anzugehen, die wir in den letzten Jahren nur in Keynotes gesehen haben.“
\end{abstract}

Marc Wagner, Managing Partner Detecon, März 2020

Bereits ohne Krise sind die Geschäftsmodelle vieler Unternehmen zunehmend im Wandel, um sich an die immer rasanteren Veränderungen der Umwelt anzupassen. Krisen wirken hier als zusätzlicher Beschleuniger. Entsprechend können Krisen und Rezessionen für viele Unternehmen und Industrien eine Zeit des tiefgreifenden Wandels bedeuten. Die wirtschaftliche Situation eines Unternehmens kann sich aufgrund des verstärkten Wettbewerbs, veränderter Beschaffungskosten, staatlicher Eingriffe oder neuer Handelspolitik ändern. Erfolgreiche Unternehmen passen ihre Geschäftsmodelle proaktiv auf diese Veränderungen an, um das bestehende Geschäft zu schützen und Vorteile zu erlangen [10]. 


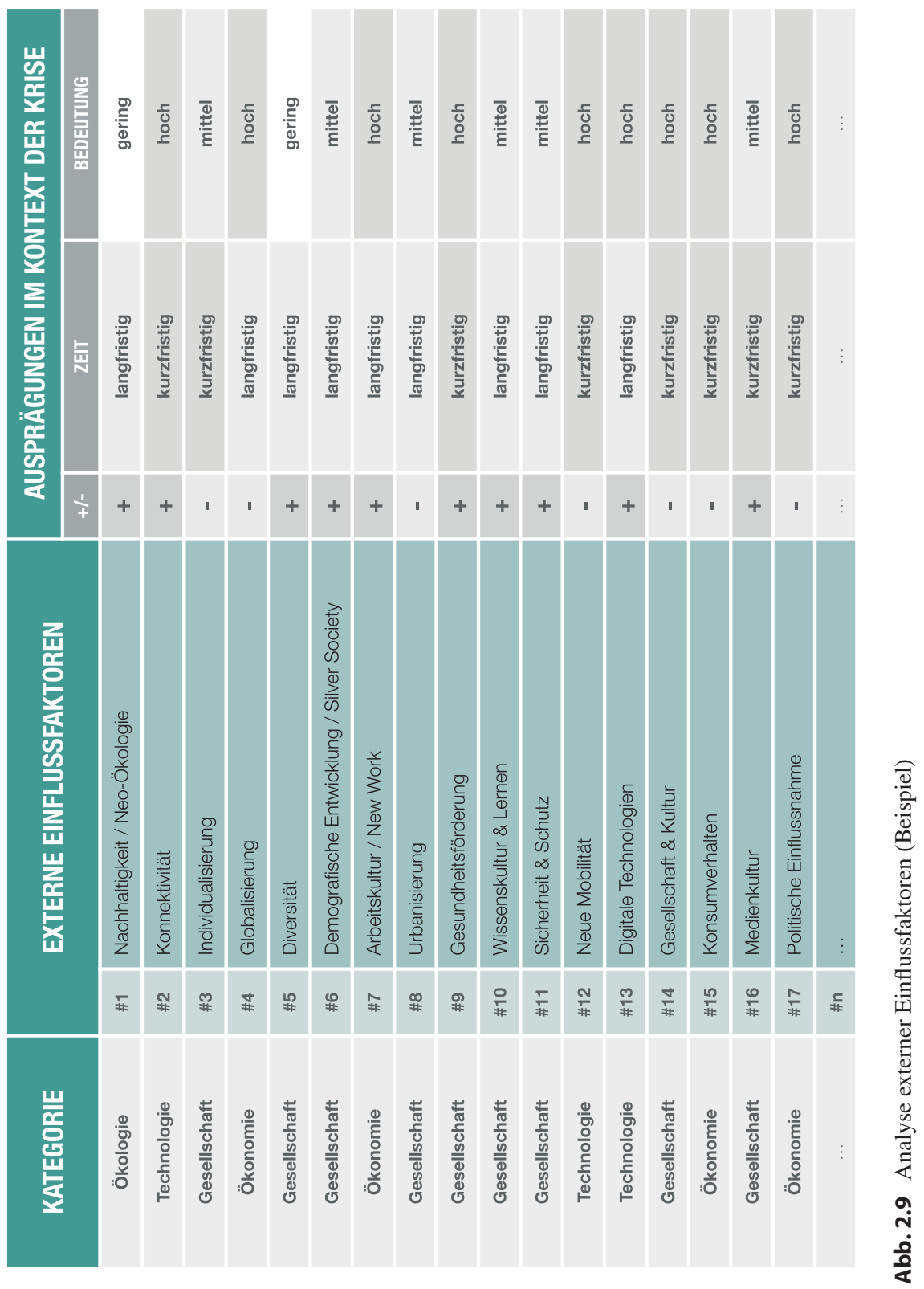




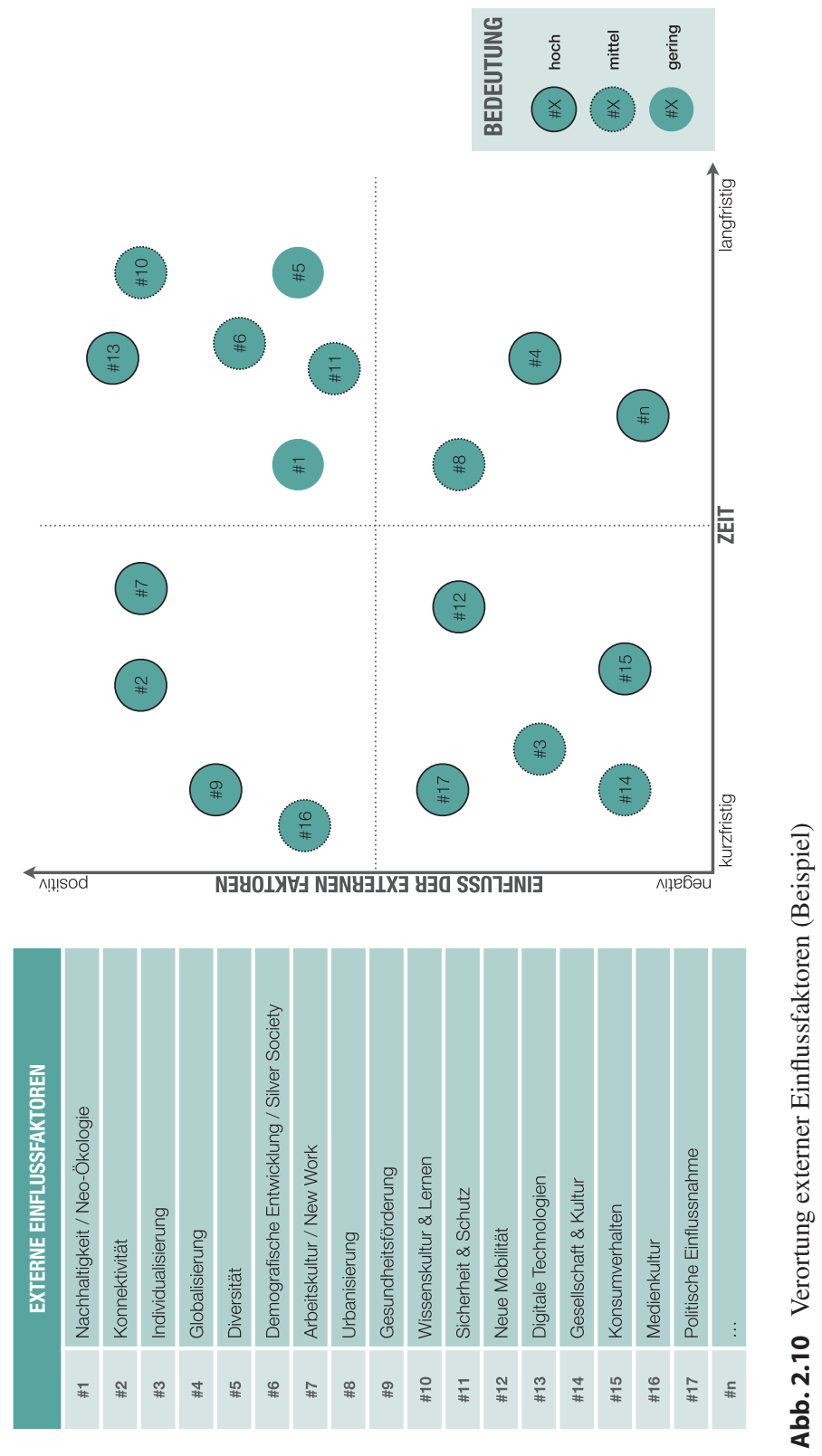




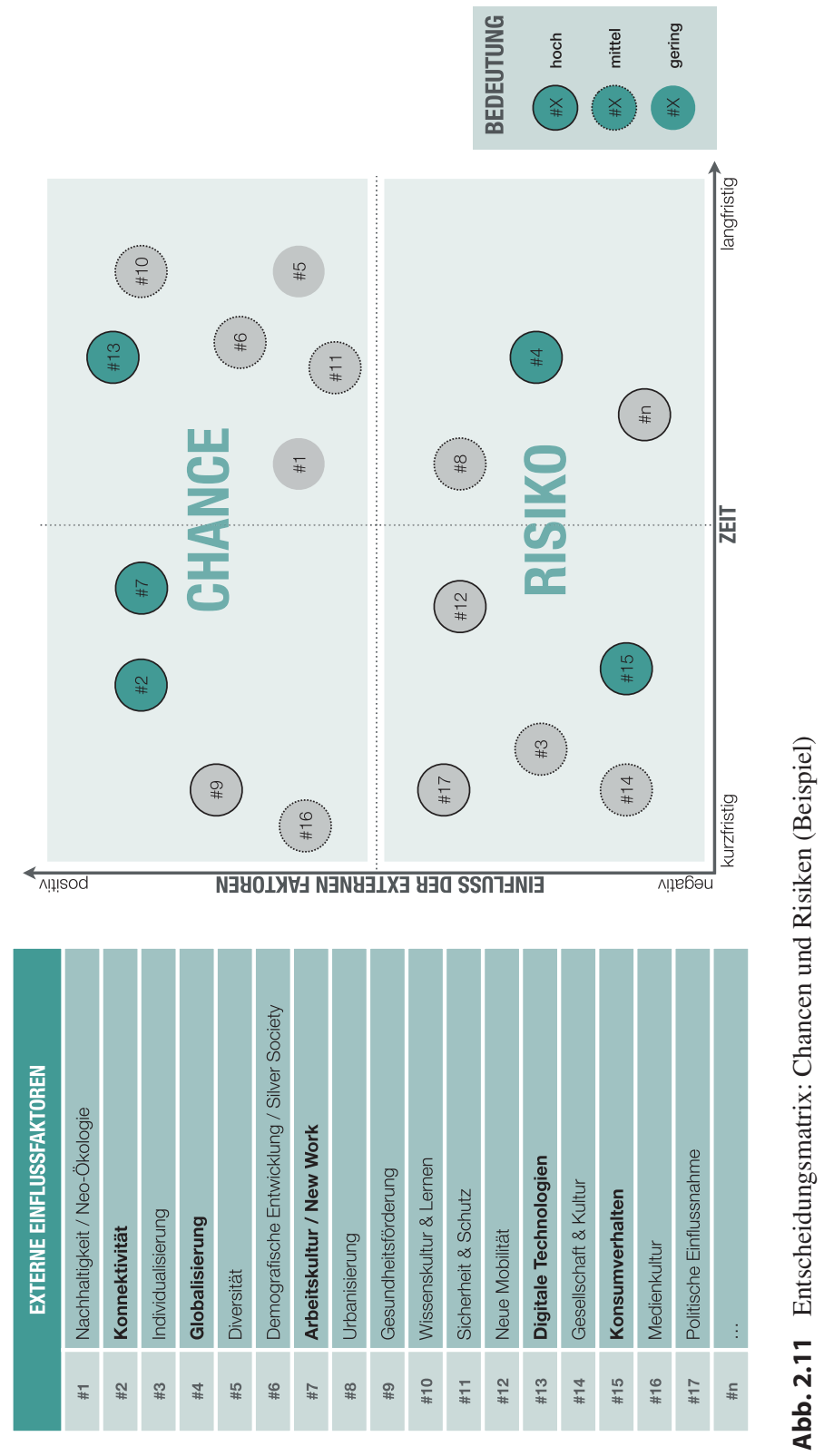


Der Einfluss von Krisen auf die Geschäftsmodelle von Unternehmen lässt sich gut an Beispielen vergangener Krisen illustrieren. In der US-Rezession der 90er Jahre verzeichnete beispielsweise IBM erstmals seit den 40er Jahren ein negatives Umsatzwachstum sowie Rekordverluste. Das Unternehmen sah sich gezwungen, sein GeschäftsmodelI zu überdenken, das damals auf dem Verkauf von Großrechnern basierte. Es erfolgte eine Verlagerung des Angebotes auf IT-Dienstleistungen und -Lösungen, mit denen IBM bis heute erfolgreich ist [12].

Im Kontext der SARS-Krise 2003 findet sich das Beispiel von Jingdong, einer kleinen Handelskette für Elektronikartikel in China. Aufgrund der mit der Krise verbundenen Einschränkungen fokussierte sich das Unternehmen darauf, das Sortiment online zur Verfügung zu stellen, was ein Jahr später in dem Onlinehändler JD.com mündete. Zwei Jahre später wurden schließlich sämtliche Filialen geschlossen und das Unternehmen wurde zu einem reinen E-Commerce Anbieter. Heute ist JD.com eine der größten E-commerce Plattformen der Welt. Auch in der Coronakrise konnte das Unternehmen bereits ein 10\%-iges Umsatzwachstum generieren [30].

Walmart konnte bisher in den USA von der Coronakrise profitieren, da Kunden in der akuten Krisensituation Vorräte anlegen und viele andere Einkaufsund Unterhaltungsmöglichkeiten geschlossen sind (Stand: April 2020). Doch neben diesen kurzfristigen Effekten plant Walmart auch von langfristigen Veränderungen zu profitieren, indem das Unternehmen die spezifischen Stärken des Geschäftsmodells wie Infrastruktur, Technologie und Personal ausspielt, um zum Beispiel verstärkt Online-Lieferungen und Marktabholungen anzubieten [31].

Aufgrund dieser Effekte ist es in Krisenzeiten wichtig, die Stärken und Schwächen des eigenen Geschäftsmodells im Kontext der veränderten Umweltsituation auf Herz und Nieren zu prüfen. Auch eine für das Unternehmen per se positive Entwicklung der Umwelt kann dabei eine Gefahr darstellen, falls das eigene Geschäftsmodell diese Entwicklung nicht antizipiert. Denn dadurch erhöht sich die Disruptionsgefahr durch bessere oder gänzlich neue Mitbewerber. Und selbstverständlich besteht bei einer aus Unternehmenssicht negativen Entwicklung des Umfeldes eine erhöhte Verlustgefahr, falls hier nicht aktiv gegengesteuert wird. Gelingt dies jedoch, kann das Unternehmen auch in einem schwierigen Umfeld wettbewerbsfähig bleiben, und von positiven Entwicklungen sogar besonders stark profitieren (Abb. 2.12).

\section{Instrument 4: Geschäftsmodell-Analyse}

Um das bestehende Geschäftsmodell systematisch zu überprüfen, werden zunächst relevante interne Faktoren im Kontext der Krisensituation identifiziert, um potenzielle Stärken und Schwächen des Unternehmens aufzudecken. 


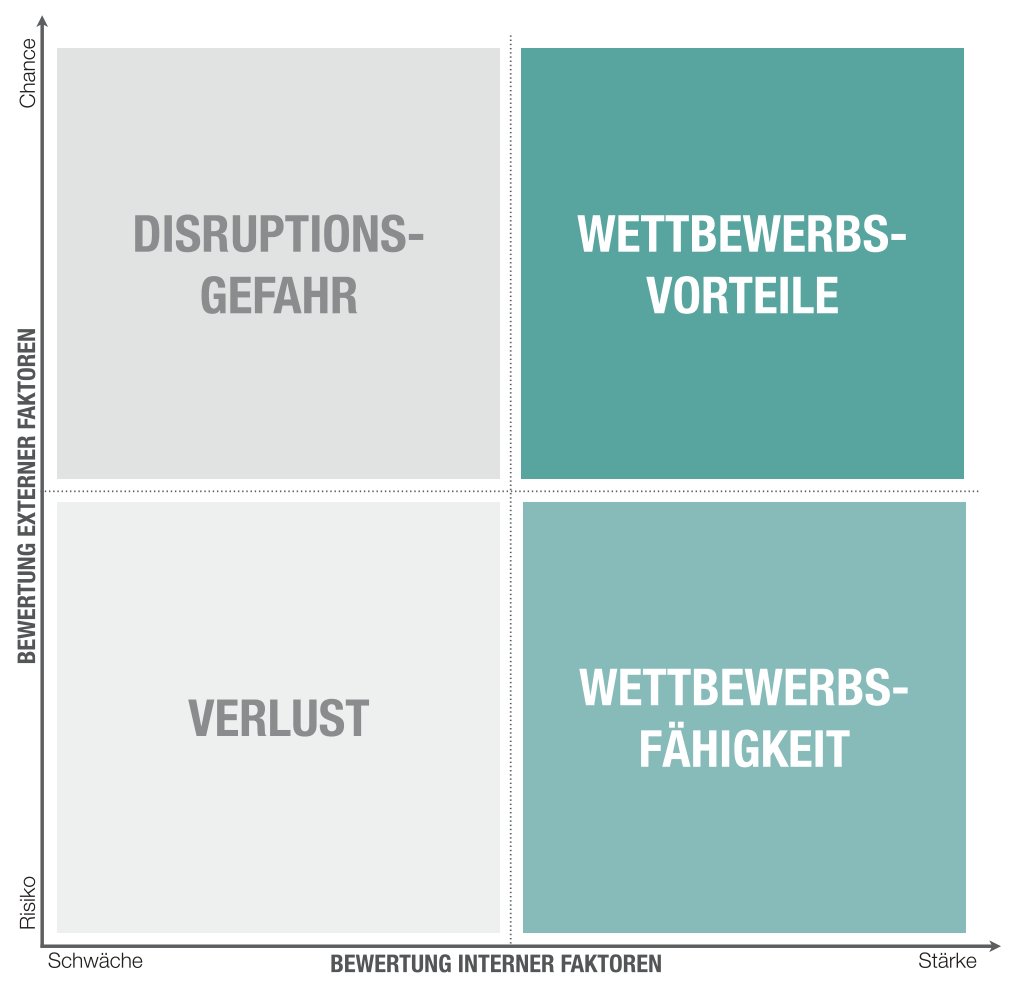

Abb. 2.12 Zusammenhang interner und externer Faktoren

Auf dieser Basis können anschließend potenzielle strategische Handlungsfelder in Bezug auf die einzelnen Aspekte des Geschäftsmodells wie beispielsweise das eigene Leistungsangebot, bestehende Vertriebskanäle oder Kernprozesse abgeleitet werden. Das Vorgehen gliedert sich dabei in drei Schritte:

\section{Identifikation relevanter interner Faktoren}

Zur Erfassung relevanter interner Faktoren im Geschäftsmodell bietet sich beispielsweise der Business Model Canvas an. Das Strategietool von Alexander Osterwalder ermöglicht eine übersichtliche Darstellung über die einzelnen Aspekte eines Geschäftsmodells und kann somit als einfache Grundlage der Geschäftsmodellanalyse dienen (Abb. 2.13). Die einzelnen Felder bilden sowohl die Marktseite (Angebot/Kundenbeziehung/Kanäle/Kunden/ 


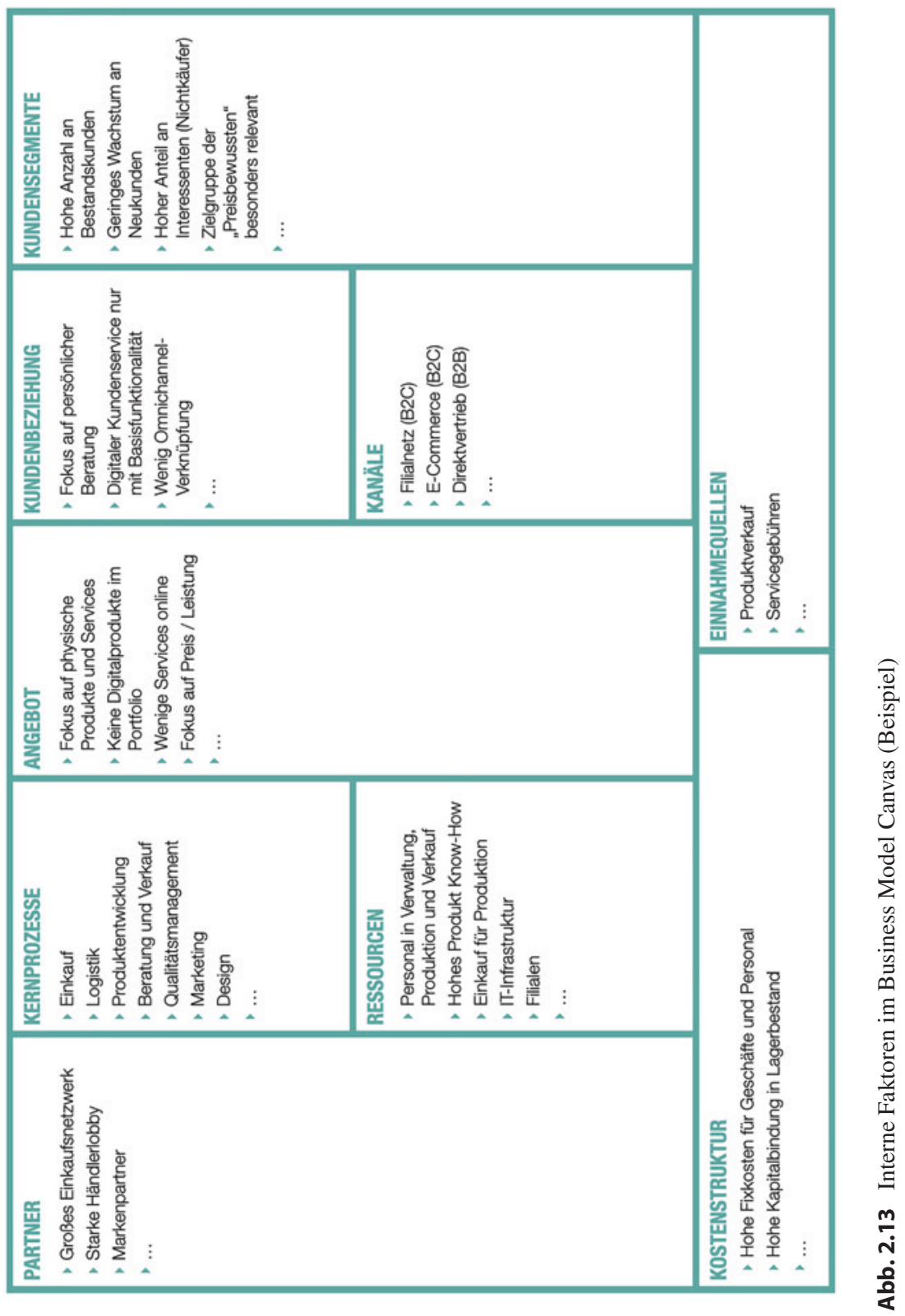


Monetarisierung), als auch die Produktionsseite (Kernprozesse/Ressourcen/ Kosten/Partner) ab. Feld für Feld können hier relevante interne Faktoren vor dem Hintergrund der Krisensituation identifiziert und dokumentiert werden. Welche Vertriebskanäle stehen mir zur Verfügung? Auf welche Lieferanten kann ich zurückgreifen? Welche Kernprozesse sind überlebenswichtig? Um vollständige Antworten zu solchen Fragen in allen Feldern des Business Model Canvas zu erhalten, sollte das Gespräch mit Führungskräften aus sämtlichen betroffenen Bereichen (Produktion, Marketing, Finanzen, etc.) gesucht werden.

2. Bewertung und Verortung relevanter interner Faktoren

Auf Basis der identifizierten, relevanten internen Faktoren gilt es nun, konkrete Stärken und Schwächen des bestehenden Geschäftsmodells in der aktuellen und (erwarteten) zukünftigen Situation abzuleiten. Dazu empfiehlt es sich, die internen Faktoren aus dem Business Model Canvas zunächst in einer Liste zu sammeln und dann in Bezug auf die Krisensituation als Stäke oder Schwäche (+/-) zu bewerten, sowie ihre zeitliche Relevanz (kurz-/langfristig) und Bedeutung (hoch/mittel/gering) in der veränderten Umwelt einzuschätzen. (Abb. 2.14).

Zur einfachen Übersicht können die internen Faktoren entsprechend ihrer jeweiligen Bewertung in eine Auswahlmatrix übertragen werden. Die Umrandung kann dabei die Bedeutung des einzelnen Faktors anzeigen, während die Position in der Matrix dem zeitlichen Horizont sowie der Kategorisierung als Stärke oder Schwäche entspricht. So wird auf einen Blick deutlich, welche bedeutsamen Stärken und Schwächen das bestehende

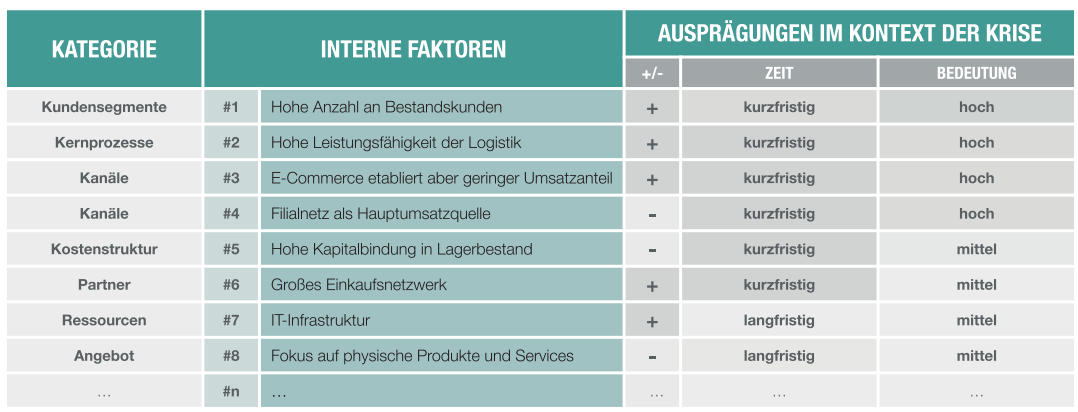

Abb. 2.14 Bewertung interner Faktoren (Beispiel) 
Geschäftsmodell im Hinblick auf die veränderte Umwelt kurz- und langfristig mit sich bringt (Abb. 2.15).

3. Auswahl von Stärken und Schwächen zur Strategieentwicklung

Nun gilt es, die relevantesten kurz- und langfristigen Stärken und Schwächen aus der Übersicht zur weiteren Bearbeitung auszuwählen. Die Auswahl erfolgt dabei entsprechend der Bewertung und Bedeutung der einzelnen internen Faktoren sowie ggf. ergänzender Diskussionen mit Entscheidungsträgern und Experten (Abb. 2.16).

\title{
2.5 Strategische Planung erneuern: Neue strategische Handlungsfelder
}

\begin{abstract}
„Die Krise kann jedoch den Wettbewerbsvorteil forschungsintensiver Unternehmen vergrößern, die die Chance nutzen, ihre Marktführerschaft durch höhere Ausgaben für Innovation, Forschung und Entwicklung zu stärken. Viele der heute führenden Unternehmen wurden in der "kreativen Zerstörung" des wirtschaftlichen Abschwungs geboren oder umgewandelt. Und mehrere der heute führenden Technologieunternehmen wie Samsung Electronics oder Google haben ihre F\&E-Ausgaben während und nach der "New Economy"-Pleite 2001 stark erhöht [32].“
\end{abstract}

OECD, Juni 2009

Die oben zitierte Analyse der OECD beschreibt, was auch zahlreiche andere Studien belegen: Insbesondere in Krisenzeiten gilt es, gezielt an neuen strategischen Handlungsfeldern zu arbeiten. Eine Studie des Global Entrepreneurship Monitor im Kontext der Finanzkrise 2008 in Spanien kommt zu dem Ergebnis, dass die Identifikation von Innovationen und Opportunitäten in Krisenzeiten wichtiger ist als in Phasen des Aufschwungs [33]. Dies lässt sich auch am Beispiel von Apple illustrieren. Obwohl sich der Umsatz des Unternehmens nach dem Platzen der Dotcom-Blase im Vergleich zum Vorjahr um ganze $33 \%$ verringerte, erhöhte Apple zur gleichen Zeit seine Forschungs- und Entwicklungsausgaben um $13 \%$, und behielt dieses Niveau auch in den Folgejahren bei. Bereits im Jahr 2003 präsentierte Apple das neue Multimedia-Verwaltungsprogramm iTunes und im Folgejahr den iPod Mini und iPod Photo. Damit läutete das Unternehmen den Beginn einer steilen Wachstumsperiode ein [34].

So zeigt sich erneut: in der Krise liegen auch Chancen. Da finanzielle Mittel in einer Krisensituation meist knapp sind, müssen die strategischen Handlungsfelder jedoch gut gewählt sein. Eine erste Indikation für die richtige Auswahl liefert eine Untersuchung aus dem Jahr 2003, die auf Basis der Daten von 


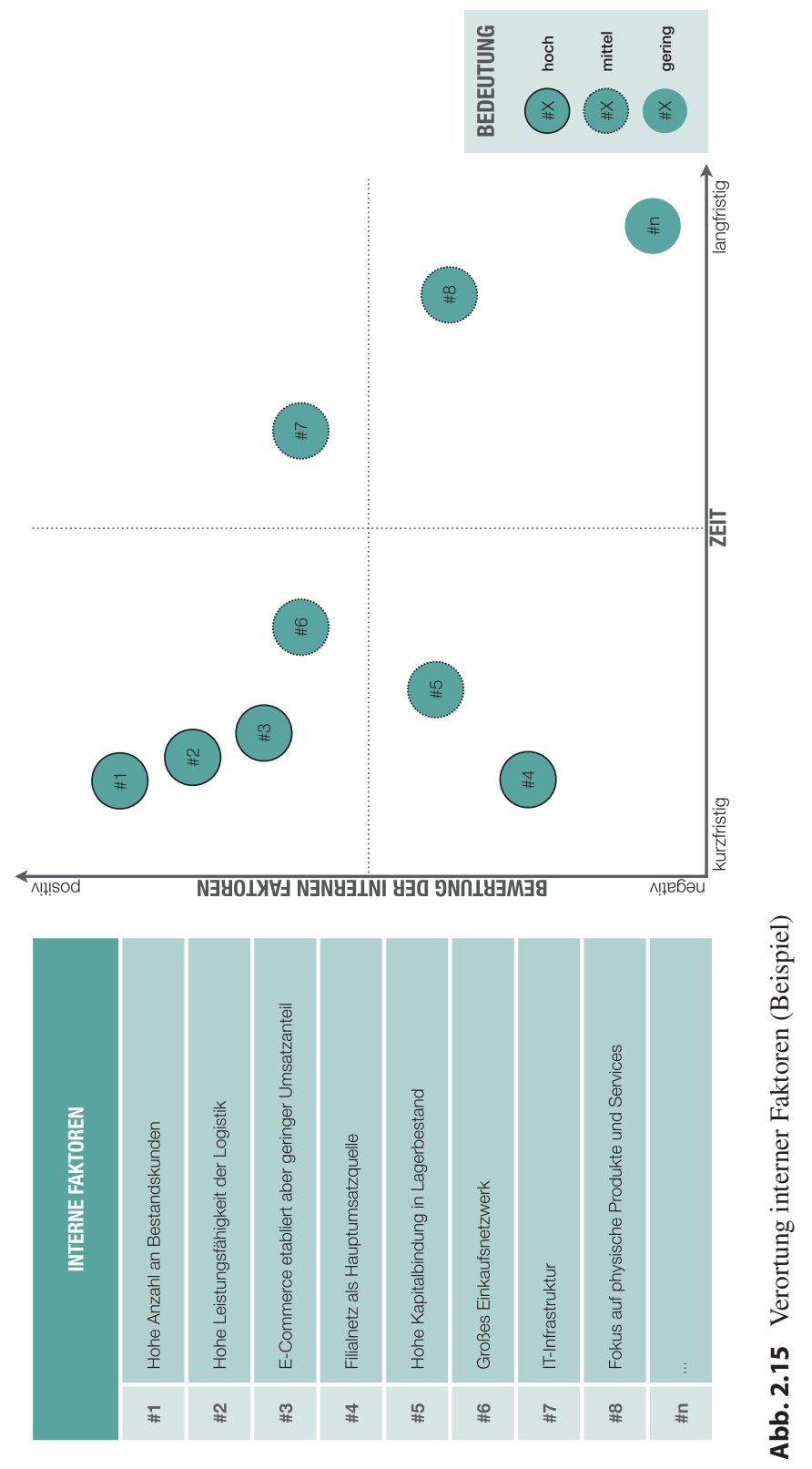




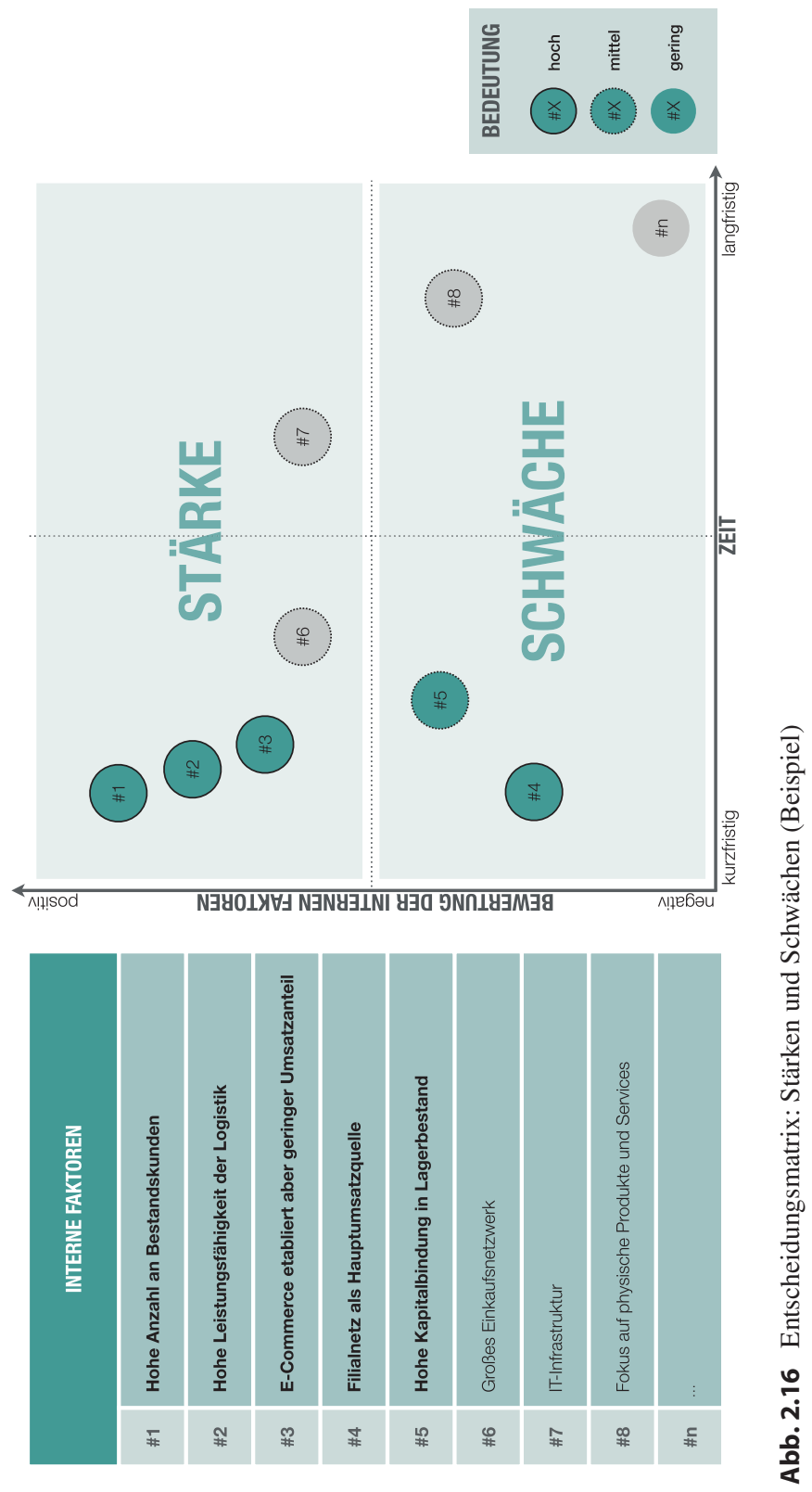


4100 Unternehmen über mehrere Rezessionen aufzeigt, dass es sich in Krisenzeiten besonders auszahlt, aggressiv in Marketing, Innovation und Kundenqualität zu investieren [35]. Gleichzeitig empfiehlt sich eine Kombination aus „Red-Ocean“-Strategien (Wettbewerb in bestehenden Märkten) und „BlueOcean"-Strategien (Schaffung neuer Märkte). Dies ermöglicht einerseits schnellen Cashflow in bestehenden Märkten zu generieren, und andererseits in neue Märkte vorzudringen, die während oder nach der Krise florieren könnten. Prominente Beispiele für den Erfolg solcher Blue-Ocean-Strategien gibt es einige, darunter erneut Apple, mit der Etablierung des Smartphones, Amazon als Pionier im Bereich des E-Commerce- und E-Book-Geschäftes, oder Netflix mit seiner erfolgreichen Streaming-Plattform [36].

\section{Instrument 5: Strategische Handlungsfelder}

Zur Ableitung neuer kurzfristiger und langfristiger Handlungsfelder für das eigene Unternehmen können die zuvor ausgewählten Einflussfaktoren, die besonders relevante Chancen, Risiken, Stärken oder Schwächen offenbart haben (Instrumente 3 und 4), zu Rate gezogen werden. Da in der Krise die finanziellen und personellen Ressourcen besonders knapp sind, ist es entscheidend, selektiv vorzugehen und einen klaren Fokus auf einige ausgewählte strategische Handlungsfelder zu legen. Auch hier erfolgt das konkrete Vorgehen in drei Schritten:

\section{Ableitung strategischer Handlungsfelder}

Den Startpunkt bilden die zuvor identifizierten und als besonders relevant bewerteten Einflussfaktoren für das Unternehmen (siehe Abschn. 2.3 und 2.4). Zur einfacheren Übersicht bietet es sich an, lediglich die ausgewählten Faktoren in der jeweiligen Matrix anzuzeigen (Abb. 2.17).

Durch die Kombination relevanter Einflussfaktoren aus der Chancen/Risikenund der Stärken/Schwächen-Matrix können nun neue strategische Handlungsfelder abgeleitet werden. (Abb. 2.18) Wachstumsstrategien entstehen in der Regel aus der Kombination ausgewählter externer Chancen mit konkreten Stärken des Unternehmens (Wettbewerbsvorteile). Abwehrstrategien lassen sich dagegen aus der Kombination externer Risiken mit bestehenden Stärken des bestehenden Geschäftsmodells ableiten (Wettbewerbsfähigkeit). Auch Anpassungsstrategien lassen sich auf diesem Wege erarbeiten. Dies geschieht, indem interne Stärken reduziert bzw. angepasst werden, um entweder externe Risiken zu adressieren (Verlustgefahr), oder Chancen nicht zu verpassen (Disruptionsgefahr) (Abb. 2.18). 


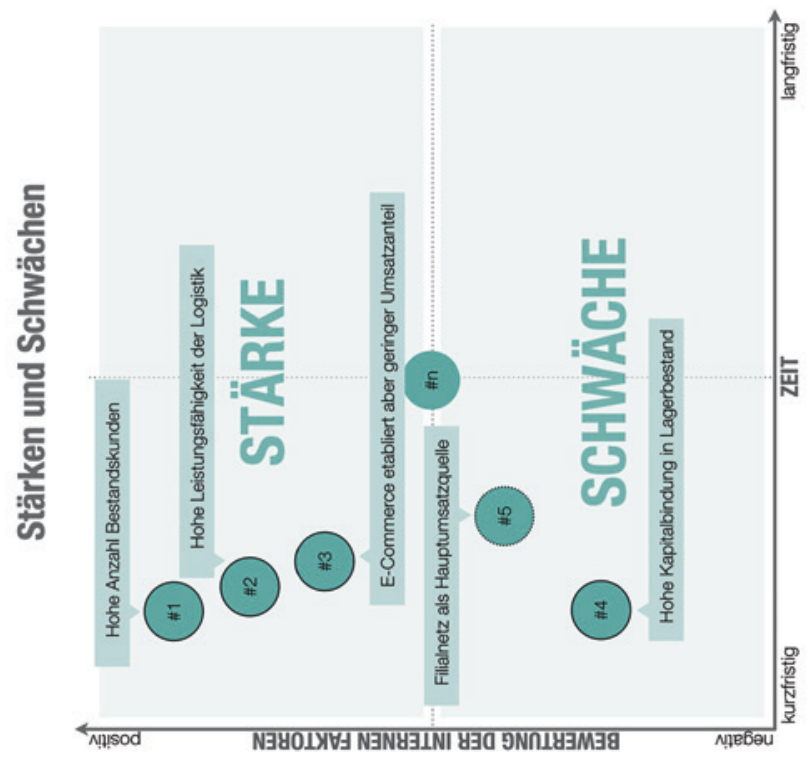

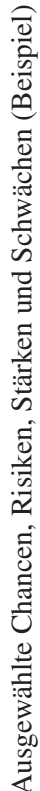
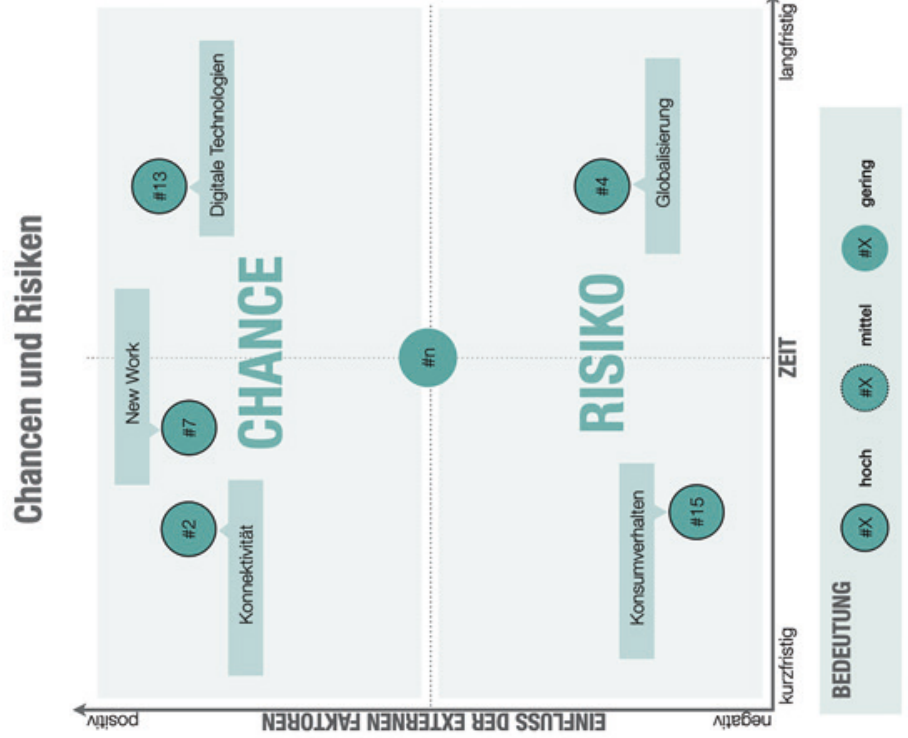


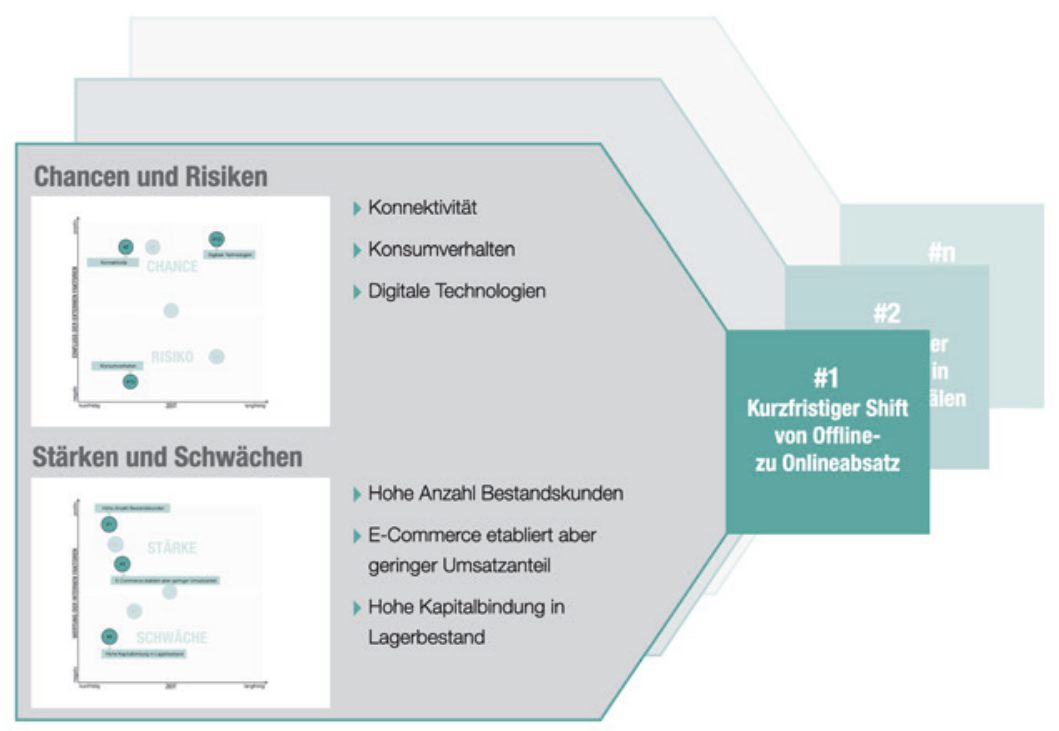

Abb. 2.18 Ableitung strategischer Handlungsfelder (Beispiel)

\section{Bewertung und Verortung strategischer Handlungsfelder}

Nachdem eine ausreichende Anzahl potenzieller kurz-, mittel- und langfristiger Handlungsfelder erarbeitet wurde, sollten diese nun bewertet werden, um eine transparente Basis für deren Auswahl zu schaffen. Auch bereits bestehende oder geplante Projekte oder Maßnahmen des Unternehmens können hier der Vollständigkeit halber mit aufgenommen werden. Alle bestehenden und neu erarbeiteten Handlungsoptionen werden zunächst in einer Liste gesammelt und in Bezug auf ihren (potenziellen) Beitrag auf die in Instrument 1 definierten Ziele und Kriterien (siehe Abschn. 2.1) bewertet (Abb. 2.19).

Eine anschließende Einordnung der strategischen Handlungsfelder in die Entscheidungsmatrix (siehe Abschn. 2.1) ermöglicht nun eine fundierte Auswahl. Dazu wird auf der Y-Achse der erwartete Beitrag des jeweiligen Handlungsfeldes auf die zuvor festgelegten kurz- und langfristigen strategischen Ziele abgetragen. Die X-Achse gibt Auskunft über die erwartete Umsetzungszeit, und damit zu einem gewissen Grad auch über Aufwand und Komplexität der verorteten Handlungsfelder. Weitere für das Unternehmen relevante Kriterien 


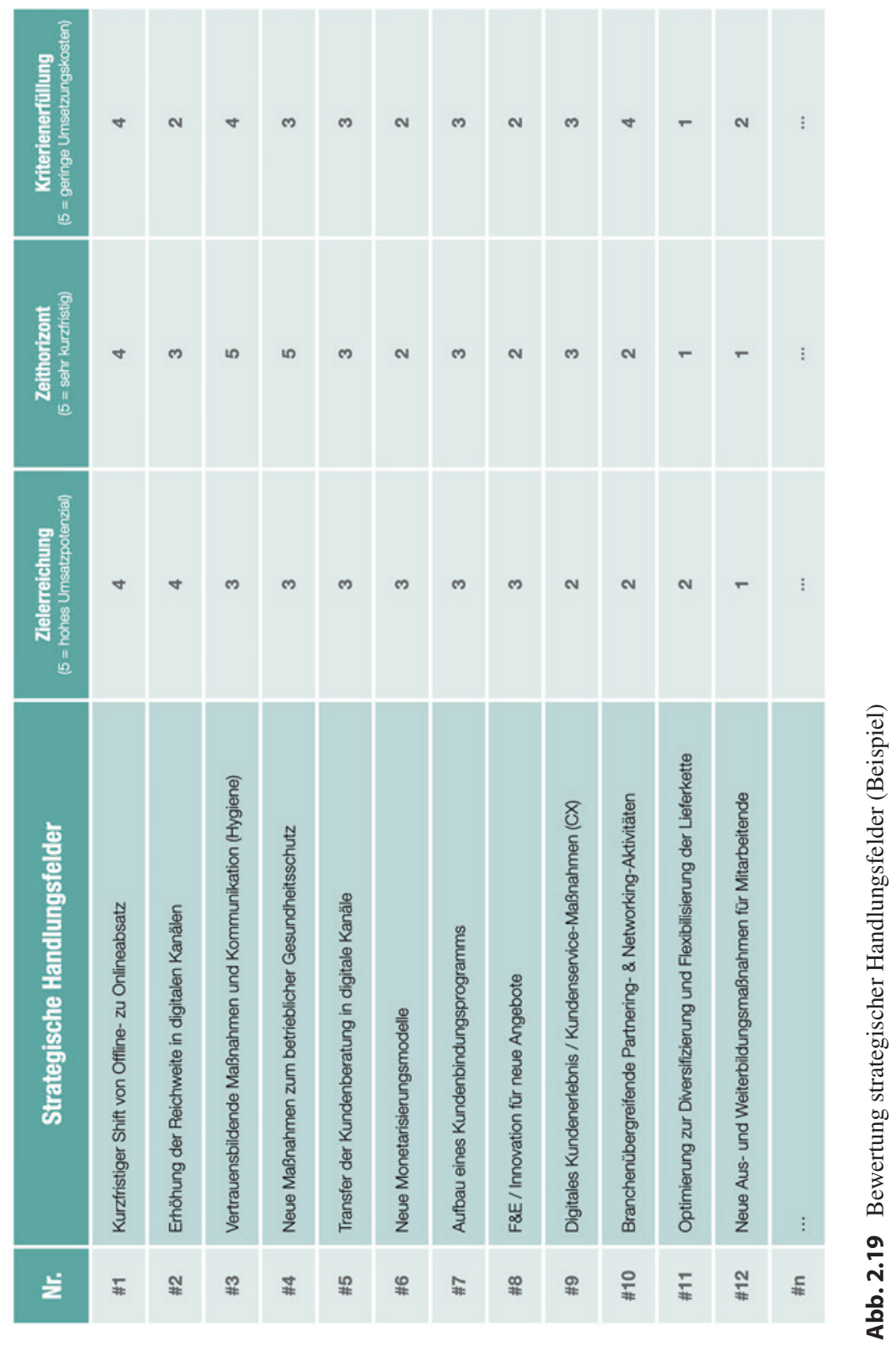


können wie bereits zuvor über die Ausgestaltung der Items, beispielsweise anhand der Umrandung der Kreise, dargestellt werden (Abb. 2.20).

3. Auswahl neuer strategischer Handlungsfelder

Bevor eine konkrete Auswahl der kurz- und langfristigen strategischen Handlungsfelder auf Basis der Entscheidungsmatrix getroffen wird, empfiehlt sich ein Rückblick auf mögliche Handlungslücken aus dem Portfoliocheck, zum Beispiel falls bislang keine ausreichende Anzahl kurzfristiger Projekte und Maßnahmen für die Bewältigung der Krise im Unternehmen vorhanden sind (siehe Abschn. 2.2). Strategische Handlungsfelder, die potenzielle "Quick Wins“ versprechen, sollten möglichst sofort angegangen werden, um das Unternehmen in der akuten Krise zu stärken (Act Now). Da jedoch auch für die (veränderte) Zukunft geplant werden muss, gilt es gleichzeitig, in strategische Wachstumsinitiativen im Sinne von „Long Term Wins“ zu investieren (Plan Now) (Abb. 2.21). Handlungsfelder, die nur begrenzt zur Erreichung der gesteckten Ziele beitragen können, sollten in Krisenzeiten möglichst gemieden werden. Ausnahme bilden hier solche Handlungsfelder, die unabhängig vom Zielbeitrag beispielsweise aus regulatorischer Sicht oder zum Schutz von Mitarbeitern und Kunden notwendig sind. Diese sollten gesondert markiert werden. Spätestens bei der Auswahl der relevanten Handlungsfelder auf Basis der Matrix sollten die Entscheidungsträger des Unternehmens intensiv eingebunden werden, um eine gemeinsame Entscheidungsbasis für die spätere strategische Roadmap sicherzustellen.

\subsection{Strategische Roadmap aufstellen: Spezifische Handlungsoptionen}

„Jetzt ist die Zeit der Unternehmer und nicht der Manager! Und dabei geht es nicht um Kategorien wie „oldschool“ oder „,newschool“. Die Buchhandlung um die Ecke muss jetzt genauso innovativ denken wie die Startups - und dabei haben keineswegs die Startups immer die besseren Karten.“

Uwe Kerkmann, Leiter der Gruppe Außenwirtschaft, Ministerium für Wirtschaft, Innovation, Digitalisierung und Energie des Landes Nordrhein-Westfalen, März 2020

In Krisenzeiten ist die Entwicklung und Bewertung strategischer Handlungsoptionen besonders schwierig und erfordert einen ausgeprägten Unternehmergeist zur strategischen Erneuerung. Schließlich bewegen sich Unternehmen hier in Zeiten großer Unsicherheit. Klassische strategische Planungsprozesse stoßen schnell an ihre Grenzen, da aufgrund der aktuellen Krise Annahmen aus der 


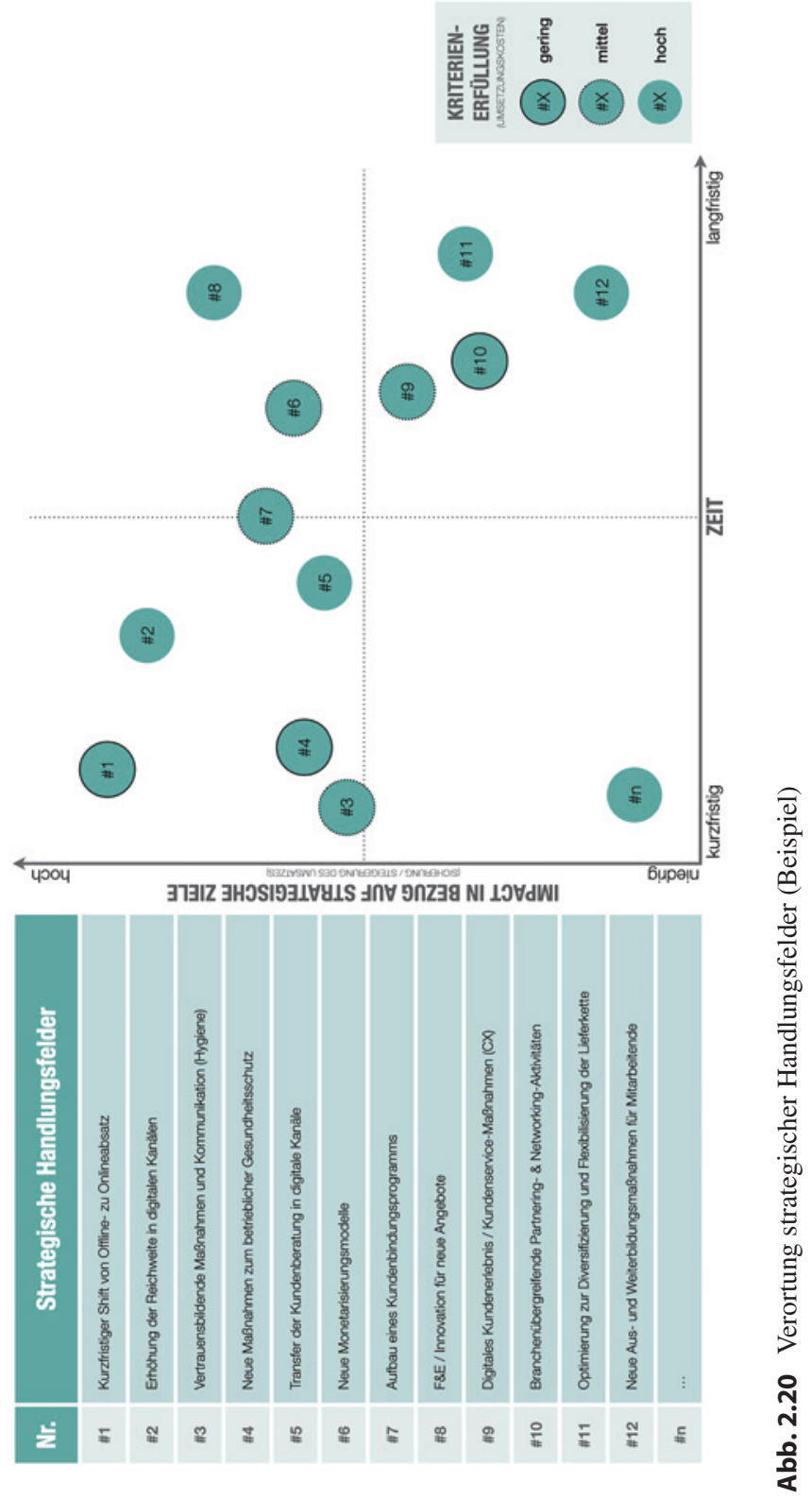




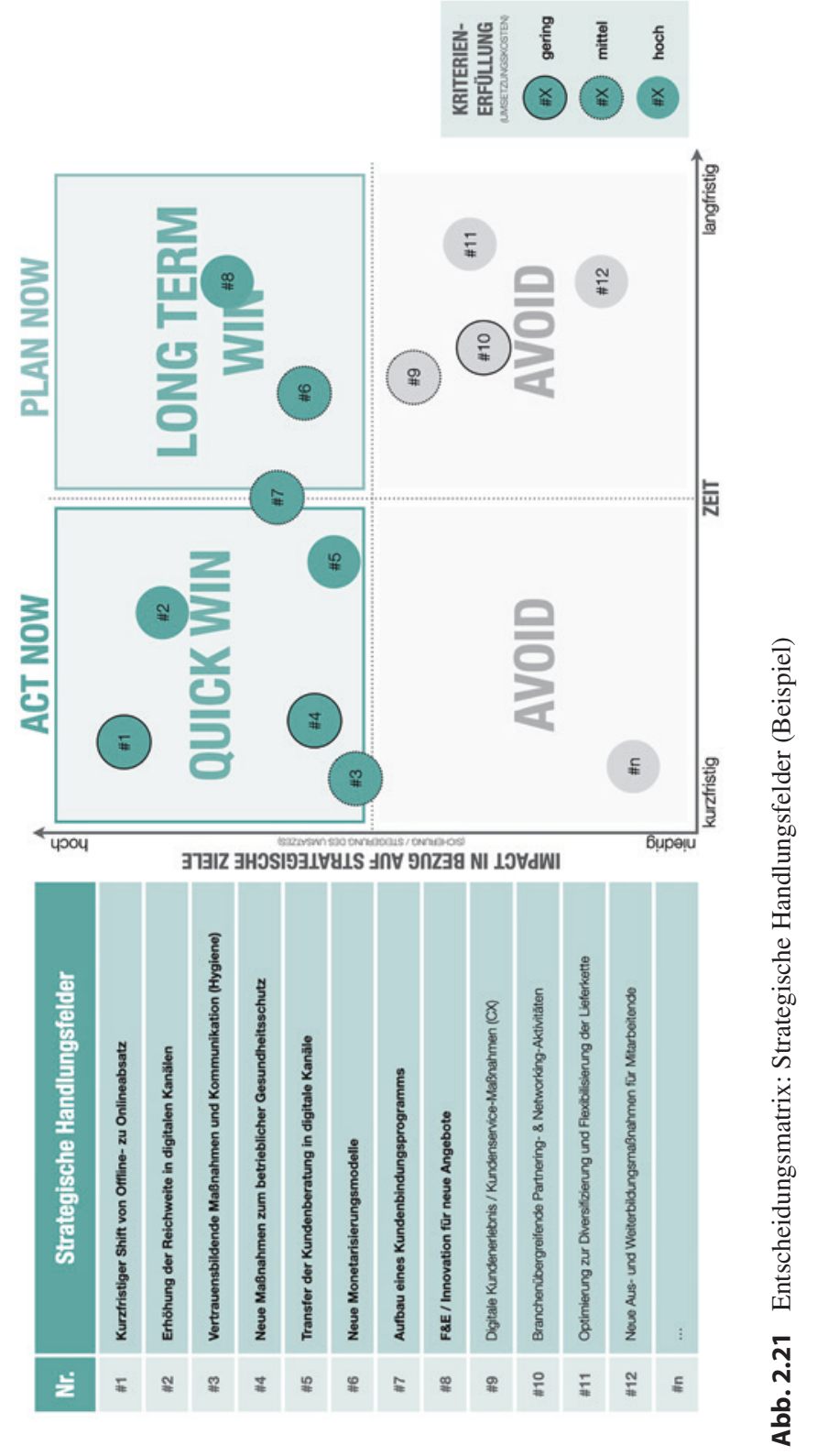


Vergangenheit in der Regel nicht einfach fortgeschrieben werden können. Selbst eine neue Erfassung der Situation, wie in den vorigen Kapiteln beschrieben, ist unter Umständen nicht ausreichend, da die Beständigkeit der identifizierten Faktoren nicht sicher vorhergesagt werden kann. Zusätzliche Szenarien helfen hier zwar, den Handlungs-Spielraum zu öffnen, bringen aber keine größere Entscheidungssicherheit mit sich, solange die einzelnen Szenarien nicht mit konkreten Wahrscheinlichkeiten versehen werden können. Am Ende bleibt stets eine gewisse Restunsicherheit.

Diese Restunsicherheit lässt sich in vier Stufen kategorisieren, wie eine Analyse der Strategy Theory Initiative zeigt. Mögliche Unsicherheits-Stufen bewegen sich laut der Analyse von „sehr gering“, im Sinne einer hinreichend klar vorhersehbaren Zukunft, über mittlere Stufen, in denen alternative Zukunftsszenarien oder eine ganze Bandbreite möglicher Zukunftsentwicklungen bestehen, bis „,sehr hoch", falls eine echte Mehrdeutigkeit vorliegt (Abb. 2.22) [37].

In Laufe einer Krise können sich Unternehmen zu verschiedenen Zeitpunkten mit unterschiedlichen Unsicherheits-Stufen konfrontiert sehen, im Extremfall auch mit einer sehr hohen Unsicherheit (echte Mehrdeutigkeit). Dies kann zum Beispiel der Fall sein, wenn eine Krise gerade akut wird und deren Ausgang und Konsequenzen noch völlig ungewiss sind. In diesem Fall herrscht eine Entwicklung, die praktisch unmöglich vorherzusagen ist. Im weiteren Krisenverlauf nimmt die Unsicherheit in der Regel wieder ab, sodass sich Entwicklungen genauer absehen lassen und die Planung entsprechend angepasst werden kann. Dennoch müssen Unternehmen auch in Situationen höchster Unsicherheit entscheidungsfähig sein, um relevante strategische Maßnahmen kurzfristig verabschieden und umsetzen zu können.

Dazu gibt es verschiedene strategische Handlungsmöglichkeiten, welche sich grob in „Big Bets“, „Optionen“ und „No-Regret“-Maßnahmen unterteilen lassen (Abb. 2.23). Big Bets wie zum Beispiel Transformationen, Firmen-Übernahmen oder neue Geschäftsfelder sind mit hohem Aufwand bzw. hohen Kosten verbunden und implizieren entsprechend auch ein erhöhtes Risiko. Optionen wie beispielsweise Pilotprojekte erlauben es, von Chancen zu profitieren und weiter zu skalieren, falls es gut läuft, ohne dass zu Beginn ein hoher Aufwand nötig ist. No-Regret-Maßnahmen wiederum haben stets einen Mehrwert für das Unternehmen und sind daher oft typische Quick Wins mit wenig Aufwand und schnellem Mehrwert wie zum Beispiel Effizienzsteigerungen oder der Aufbau neuer relevanter Fähigkeiten.

Insbesondere in einer sehr unsicheren Krisensituation ist es sinnvoll, ein Portfolio mit diversen strategischen Maßnahmen zur Hand zu haben. So kann das Unternehmen kurzfristig auf No-Regret-Maßnahmen und Optionen aufsetzen, und bei besonders relevanten Chancen auch Big Bets eingehen [37]. 

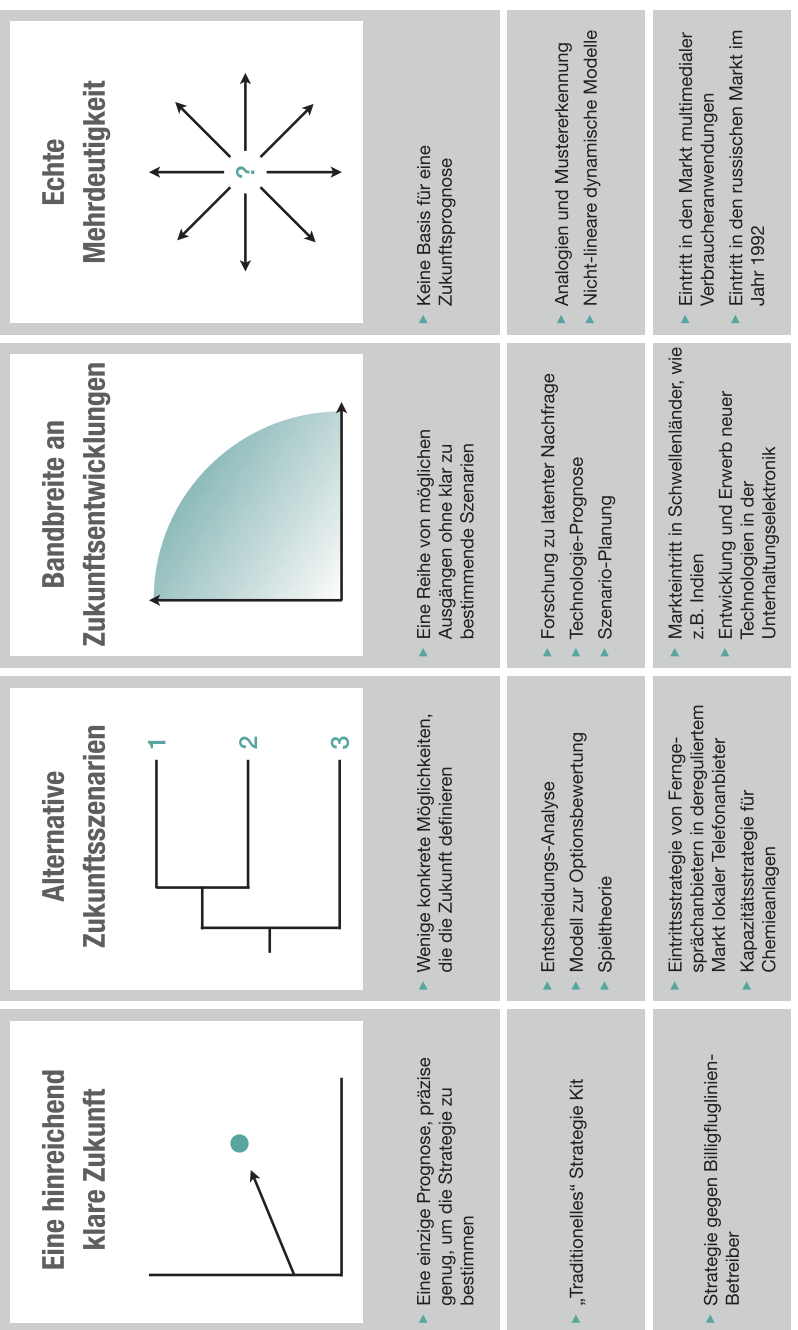

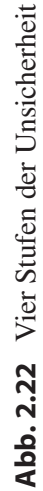




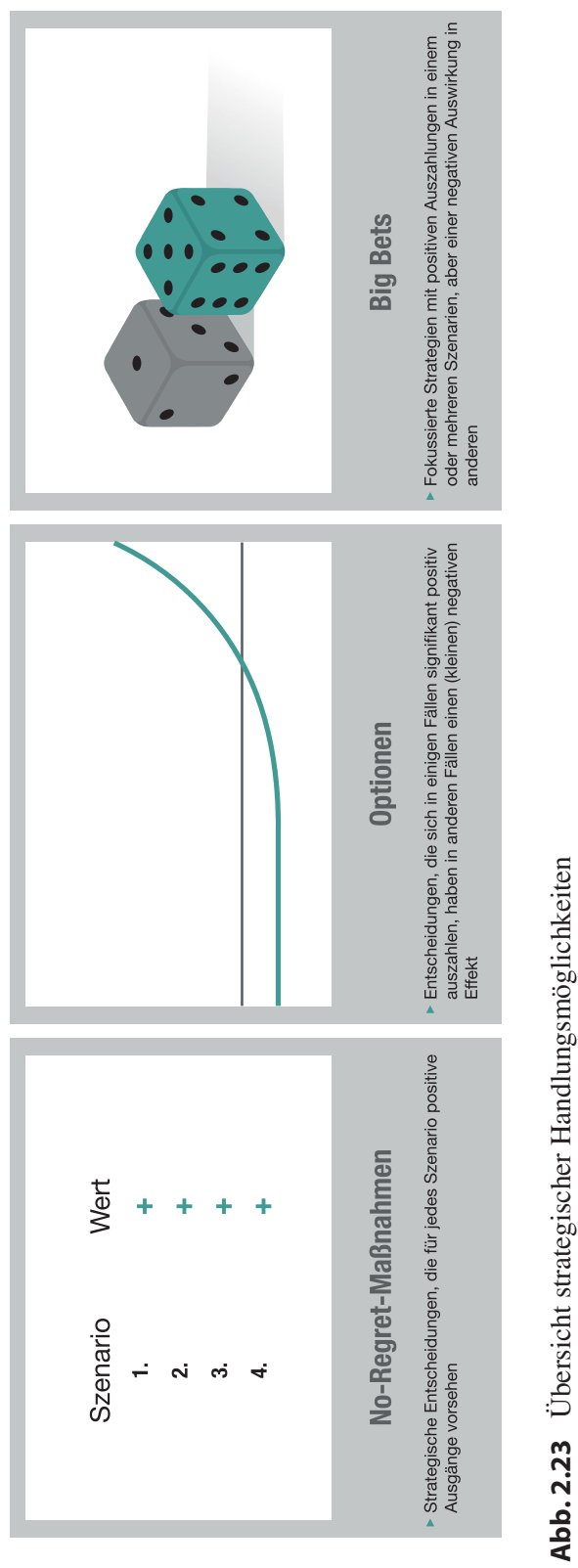


Doch welche Big Bets, Optionen oder No-Regret-Maßnahmen haben sich in Krisen bislang bewährt? Laut einer Analyse des Managementforschers Herrmann Simon bieten sich als No-Regret-Maßnahmen in Krisenzeiten zum Beispiel ungewöhnliche Garantien, eine erfolgsabhängige Bezahlung, Cross-Selling-Maßnahmen, oder auch eine Intensivierung sowie stärkere Monetarisierung von Services an. Denn mithilfe dieser Maßnahmen, so Simon, kann potenziell das Vertrauen gestärkt, der Cashflow gesichert und der Umsatz schnell gesteigert werden. $\mathrm{Zu}$ den sinnvollen Optionen im Kontext einer Krise zählen laut der Analyse insbesondere alternative Monetarisierungsmodelle, die Erschließung neuer Kundensegmente, die Ausweitung der Serviceangebote und Systemlösungen, sowie eine Erweiterung des Verriebssortiments. So können bestenfalls die Finanzen optimiert und der Umsatz nachhaltig gesteigert werden [38]. Abhängig von den individuellen Zielen des Unternehmens (siehe Abschn. 2.1) können dies bereits erste Inspirationen für mögliche Maßnahmen in den ausgewählten strategischen Handlungsfeldern sein.

In Bezug auf Big Bets kann es sinnvoll sein, auf besonders langfristige und nachhaltige Trends aufzusetzen, die möglichst unabhängig von der Krise sind. So könnten zum Beispiel Investitionen in Lösungen für den Klimaschutz getätigt werden, in der Annahme, dass der Klimawandel durch die aktuelle Krise nicht plötzlich zum Erliegen kommt und somit langfristig ein relevantes Betätigungsfeld darstellt. Solche „Zukunftsinnovationen“ können Teil der verabschiedeten strategischen Maßnahmen sein, um auch nach der Krise gut aufgestellt zu sein.

\begin{abstract}
„Neue Technologien sind der Schlüssel zur Zukunft der Mobilität. Bis 2025 nehmen wir mehr als 30 Milliarden Euro für Forschung und Entwicklung in die Hand, um unsere Rolle als Innovationsführer weiter auszubauen. Das ist auch Ausdruck unserer Zuversicht für die zukünftige Geschäftsentwicklung. Von entscheidender Bedeutung ist dabei die Fähigkeit, unterschiedlichste Technologien in ein Gesamtsystem zu integrieren: Wer Hardware und Software gleichermaßen beherrscht und zusammenführt, wird nachhaltig die Zukunft des Automobils gestalten. Wir sehen uns hier ganz klar auf der Überholspur [39].“
\end{abstract}

Oliver Zipse, CEO BMW, März 2020

\title{
Instrument 6: Strategische Handlungsoptionen
}

Um eine konkrete strategische Roadmap für die Zeit während und nach der Krise zu verabschieden, müssen die zuvor erarbeiteten und ausgewählten strategischen Handlungsfelder zunächst systematisch detailliert und analysiert werden, um Anknüpfungspunkte für konkrete Handlungsoptionen aufzudecken und spezifische Projekte und Maßnahmen abzuleiten. Anschließend können diese bewertet 
und je nach Relevanz zur kurz- oder langfristigen Umsetzung ausgewählt und als Roadmap dargestellt werden. Das konkrete Vorgehen gliedert sich dazu in drei Schritte:

\section{Systematische Detaillierung der ausgewählten Handlungsfelder}

Basis für die Erarbeitung konkreter Handlungsoptionen sind die zuvor ausgewählten strategischen Handlungsfelder. Diese gilt es nun, systematisch $\mathrm{zu}$ detaillieren, um möglichst viele potenzielle Anknüpfungspunkte für spezifische Projekte oder Maßnahmen aufzudecken. Dazu bietet sich der sogenannte morphologische Kasten an, mit dessen Struktur das jeweilige Handlungsfeld zunächst in einzelne Parameter aufgegliedert wird, die dann wiederum in möglichst viele Ausprägungen spezifiziert werden. Ein Handlungsfeld könnte beispielsweise in einzelne Parameter wie „Zielgruppen“, „Kanäle“ oder „Monetarisierungsmöglichkeiten“ aufgegliedert werden. Und ein Parameter wie „Zielgruppen“ dann wiederum in einzelne Ausprägungen wie „B2B“, „B2C“, „Frauen“, „Männer“, „Preisbewusste“, etc. spezifiziert werden (Abb. 2.24). Die Materialisierung des morphologischen Kastens kann zum Beispiel mithilfe einer einfachen Excel-Tabelle erfolgen.

2. Detaillierte Analyse zur Ableitung von Handlungsoptionen

Sobald ein Handlungsfeld als morphologischer Kasten in Parameter und Ausprägungen spezifiziert wurde, ist im nächsten Schritt eine detaillierte Sekundärforschung vonnöten. Es gilt, die einzelnen Parameter und Ausprägungen beispielsweise mit Hilfe von vorhandenen Studien, Trends, Befragungen oder internen Marktforschungsdaten zu analysieren, um möglichst viele konkrete Ansatzpunkte für spezifische Projekte oder Maßnahmen innerhalb des Handlungsfeldes aufzudecken (Abb. 2.25). Abhängig vom zu untersuchenden Handlungsfeld kann es sinnvoll sein, neben den sekundären auch primäre Forschungsaktivitäten wie zum Beispiel Expertenbefragungen oder eigene Umfragen durchzuführen. Durch die detaillierte Analyse in diesem Schritt können nicht nur besonders relevante, spezifische Potenziale innerhalb eines Handlungsfeldes aufgedeckt werden. Durch die Fundierung eines Ansatzpunktes mit Hilfe von Fakten aus Sekundär- und ggf. Primärforschung ist auch die nötige Basis für eine möglichst objektive Bewertung der abgeleiteten Projekte und Maßnahmen gegeben (Abb. 2.26).

3. Bewertung und Auswahl strategischer Handlungsoptionen

Die aus der Analyse abgeleiteten strategischen Handlungsoptionen, im Sinne von spezifischen Projekten und Maßnahmen, gilt es nun, in Bezug auf ihren (potenziellen) Beitrag auf die vorher definierten Ziele und Kriterien (siehe Abschn. 2.1) zu bewerten. Eine anschließende Einordnung in die Ent- 


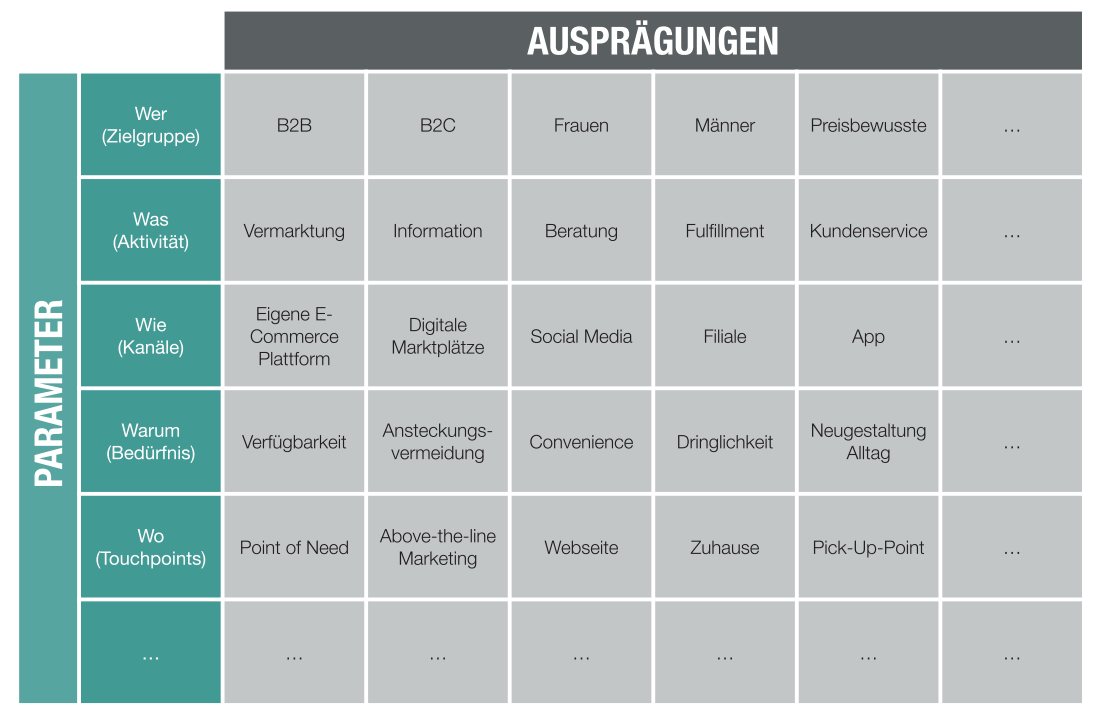

Abb. 2.24 Übersicht eines morphologischen Kastens (Beispiel)

scheidungsmatrix ermöglicht eine transparente, visuelle Darstellung aller Handlungsoptionen als Portfolio. Dazu wird auf der Y-Achse der erwartete Beitrag der jeweiligen Handlungsoption auf die kurz- und langfristigen strategischen Ziele abgetragen. Die X-Achse gibt wie gewohnt Auskunft über die erwartete Umsetzungszeit bzw. indirekt auch über Aufwand und Komplexität der verorteten Handlungsoptionen. Relevante Kriterien können erneut durch die unterschiedliche Ausgestaltung der einzelnen Items dargestellt werden (Abb. 2.27).

Nun ist die Voraussetzung für eine finale Auswahl umzusetzender Projekte und Maßnahmen geschaffen. In Krisenzeiten gilt es zunächst, eine ausreichende Anzahl von kurzfristigen Quick Wins sicherzustellen, darunter bestenfalls möglichst viele No-Regret-Maßnahmen. Zudem sollten für die mittel- und langfristige Planung auch Optionen mit ungewisserem Impact und geringem Aufwand, sowie Big Bets mit hohem Aufwand, aber gleichzeitig hohem potenziellen Zielbeitrag in das Strategieportfolio aufgenommen werden. So wird eine passende Balance zwischen Projekten und Maßnahmen für die Zeit während und nach der Krise sichergestellt. Handlungsoptionen, die einen geringen potenziellen Zielbeitrag in Verbindung mit einem hohen Aufwand 


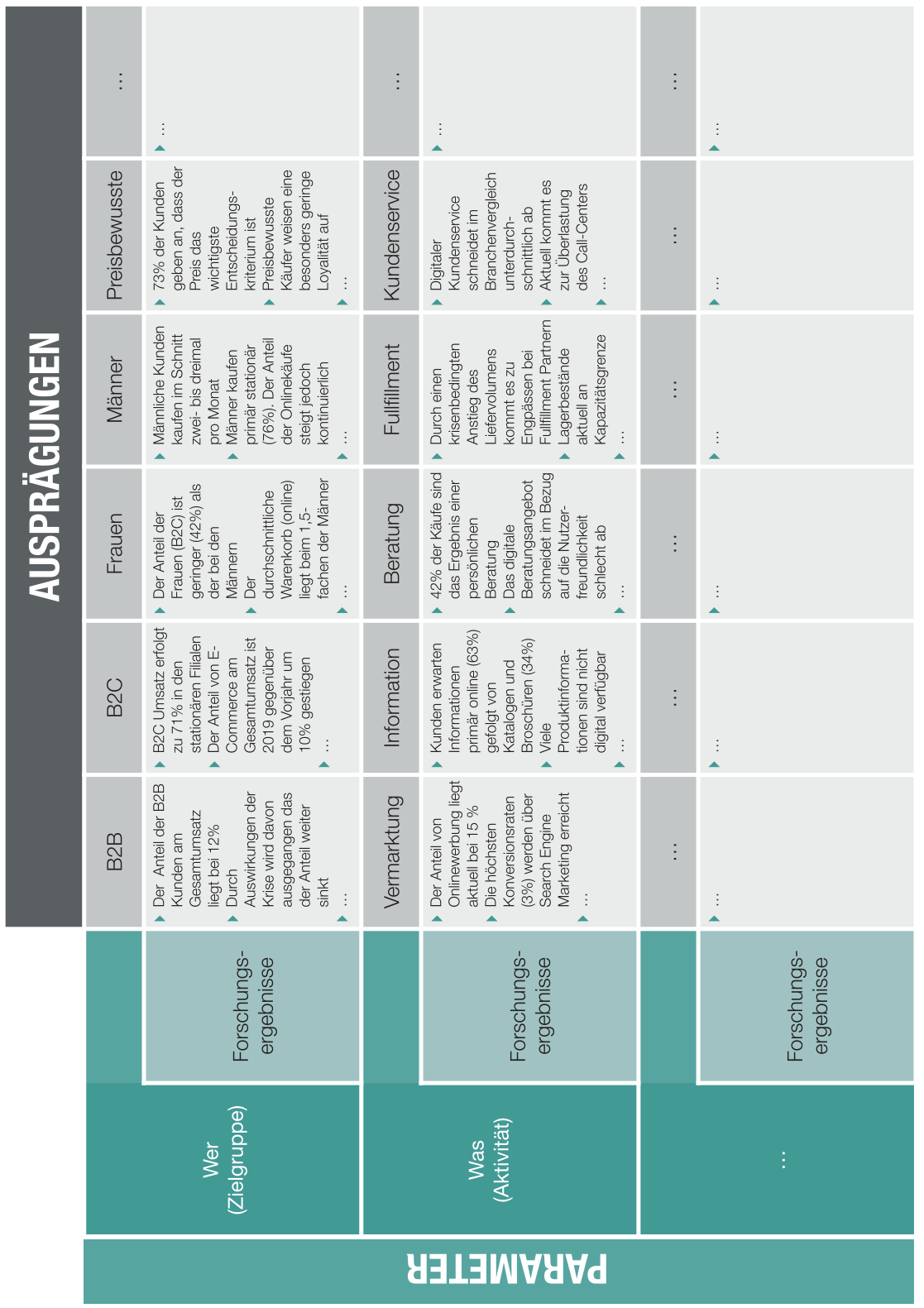




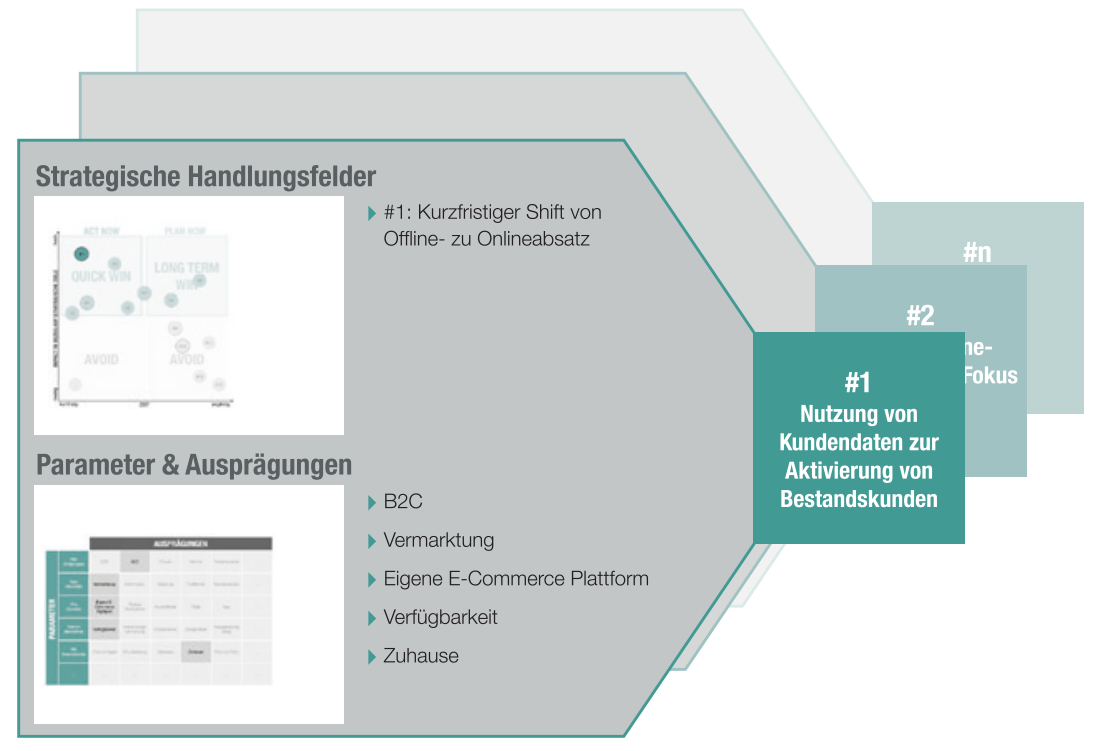

Abb. 2.26 Ableitung von Handlungsoptionen (Beispiel)

aufweisen, sollten grundsätzlich vermieden werden, es sei denn, diese sind unabhängig vom Zielbeitrag, zum Beispiel aus rechtlichen Gründen, für das Unternehmen von Bedeutung. In diesem Fall sollten diese gesondert markiert werden (Abb. 2.28).

Für die weitere Konkretisierung der Umsetzungsplanung empfiehlt es sich, die ausgewählten Handlungsoptionen in eine Roadmap zu übertragen. Wann ein bestimmtes Projekt oder eine bestimmte Maßnahme umgesetzt werden soll bzw. kann, hängt dabei nicht nur von dessen spezifischer Komplexität ab, sondern auch von der Verfügbarkeit von internen oder externen Ressourcen, sowie möglichen Abhängigkeiten zwischen einzelnen Maßnahmen. Dies gilt es bei der Planung zu berücksichtigen (Abb. 2.29). Die Roadmap kann im Gegensatz zu einem Projektplan auch dynamisch verstanden werden. Entsprechend können Zeiträume unter Umständen noch verändert werden, wenn im Zuge der weiteren Ausarbeitung der einzelnen Projekte und Maßnahmen ein tieferes Detail- und Planungslevel erreicht wird. 


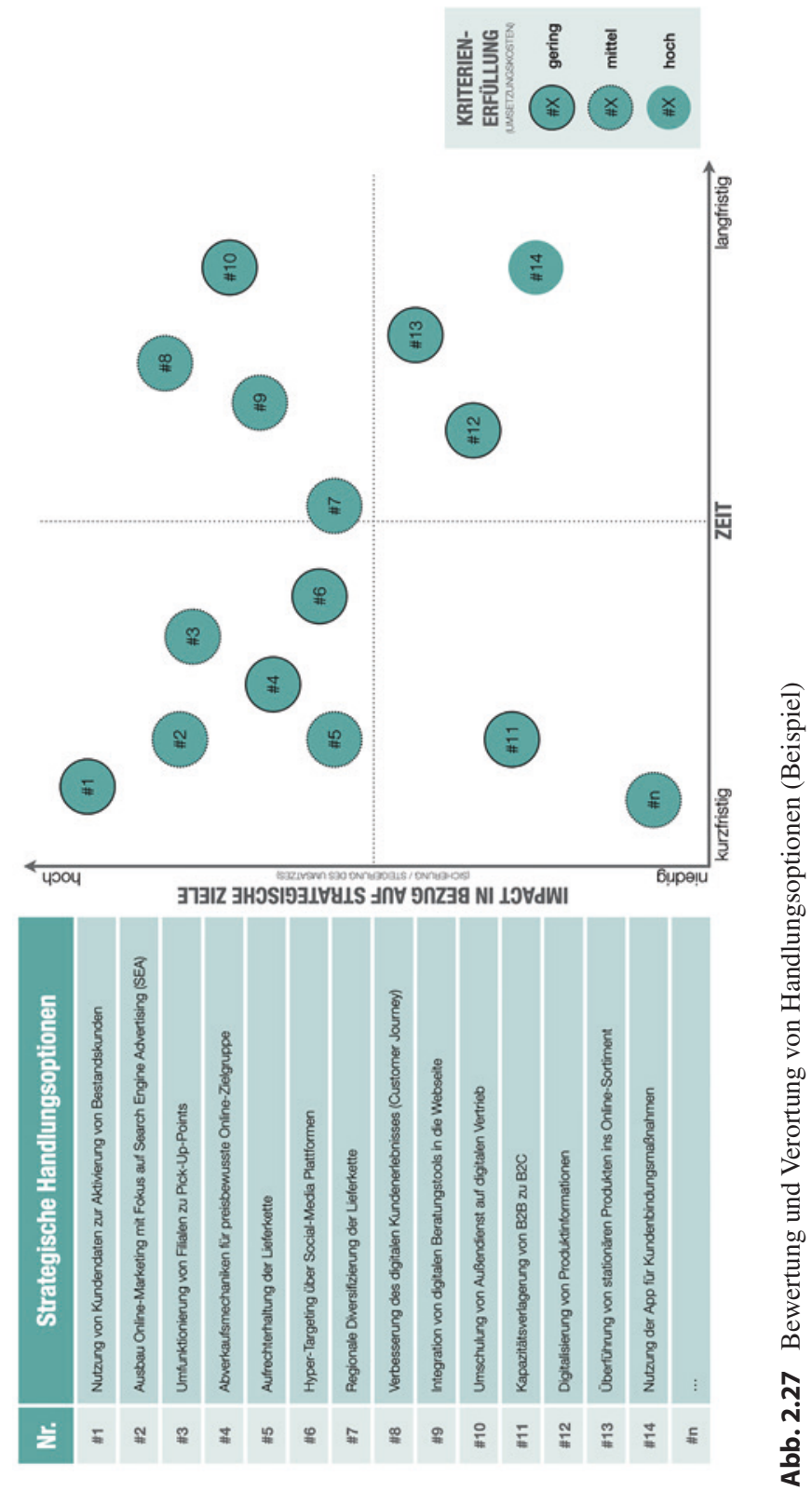



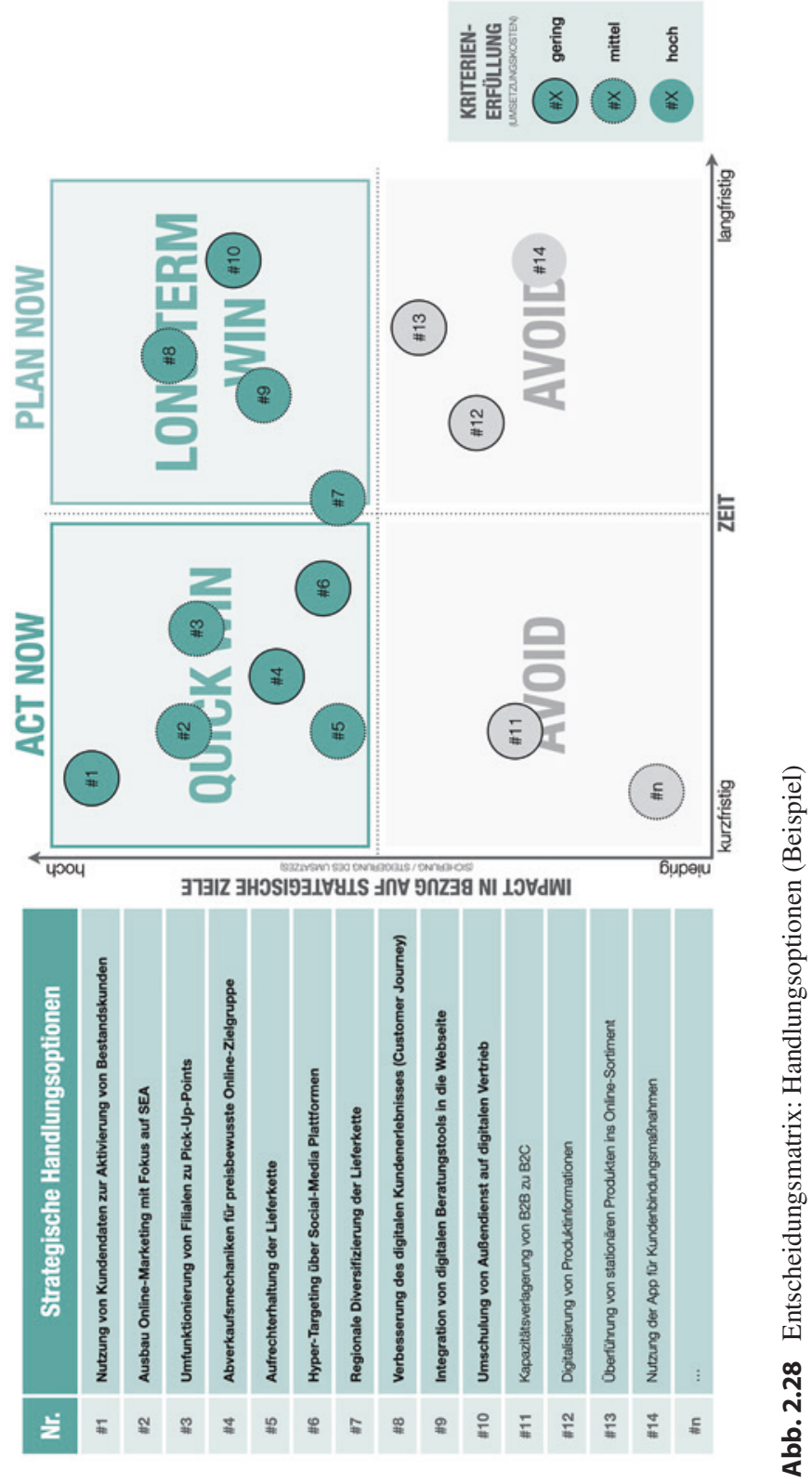


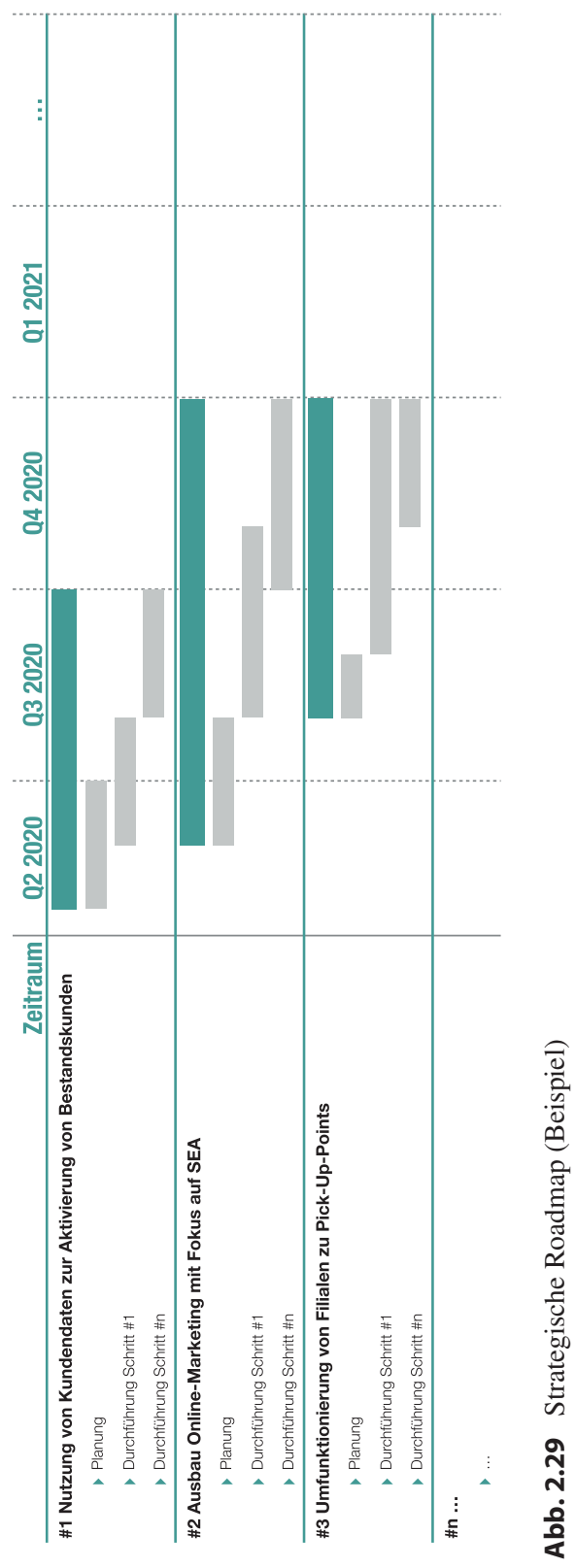




\subsection{Kontinuierliche Anpassung etablieren: Dynamischer Strategieprozess}

"Im Moment ist eine verlässliche Prognose schlicht nicht möglich. Schwere und

Dauer der Krise sind aktuell nicht einzuschätzen [40].“

Frank Witter, Finanzvorstand Volkswagen, März 2020

In einer relativ stabilen und vorhersehbaren Welt dienen Strategien dazu, einen möglichst dauerhaften, und somit implizit statischen Wettbewerbsvorteil aufzubauen. Doch in Krisenzeiten können bisherige Wettbewerbsvorteile plötzlich zu Nachteilen werden und Marktanteile können sich schnell verschieben [4]. Im Kontext einer Krise kommt es nicht nur darauf an, die richtigen strategischen Maßnahmen zu definieren und umzusetzen. Auch sogenannte dynamische Fähigkeiten sind entscheidend, um eine schnelle Adaption und Veränderung zu ermöglichen. Schließlich gilt es in einer Krise, strategische Entscheidungen kontinuierlich auf den Prüfstand zu stellen: Sind die gewählten Ziele und Kriterien noch passend? Wie laufen die bestehenden Projekte? Haben sich externe Einflussfaktoren geändert, und zeigen sich dadurch neue Stärken und Schwächen im Geschäftsmodell?

Um diese und andere strategische Fragen während einer Krise bestenfalls in einem monatlichen oder gar wöchentlichen Turnus zu klären, gilt es, die Entscheidungsfindung im Unternehmen signifikant $\mathrm{zu}$ beschleunigen. Die im Rahmen des beschriebenen Strategieprozesses erarbeitete Entscheidungsmatrix bietet dazu die nötige Voraussetzung. Bei neuen Erkenntnissen in Bezug auf die Krise können sowohl die gewählten Ziele und Kriterien, als auch die definierten Handlungsfelder bzw. -optionen schnell und effizient in der Entscheidungsmatrix angepasst und ggf. sogar neue Projekte oder Maßnahmen definiert werden (Abb. 2.30). Das Instrument wird dadurch zu einer ,agilen Strategiematrix“, die in jeder strategischen Diskussion als klar strukturierte, zielgerichtete und transparente Diskussionsgrundlage dienen kann.

Mithilfe der Strategiematrix sowie des gesamten in Kap. 2 skizzierten Strategieprozesses (Abb. 2.31), wird es für Unternehmen möglich, in Krisenzeiten schnell und proaktiv zu agieren und somit nicht nur erfolgreich durch die Krise zu kommen, sondern bestenfalls auch durch die Krise erfolgreicher zu werden.

Für Leser dieses Buches stellen wir wichtige Instrumente zur Strategieentwicklung in Krisenzeiten auch digital zur Verfügung. Diese können Sie auf folgender Webseite abrufen: www.erfolgreich-durch-die-krise.com. 


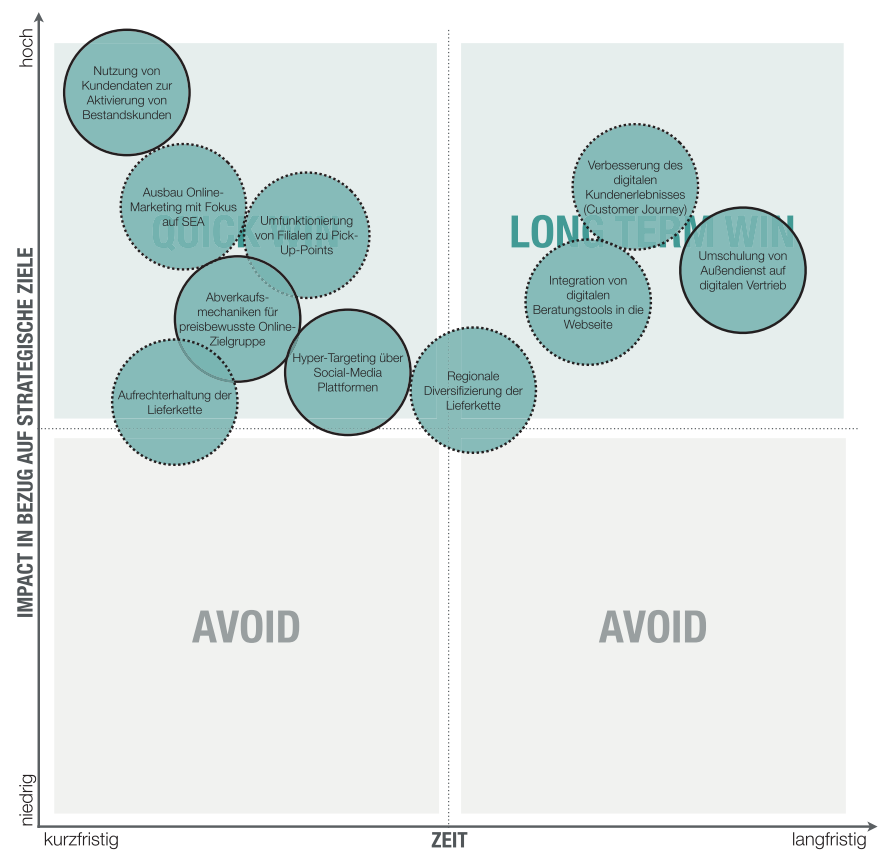

KRITERIENERFÜLLUNG

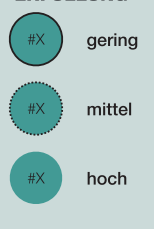

Abb. 2.30 Agile Strategiematrix (Beispiel)

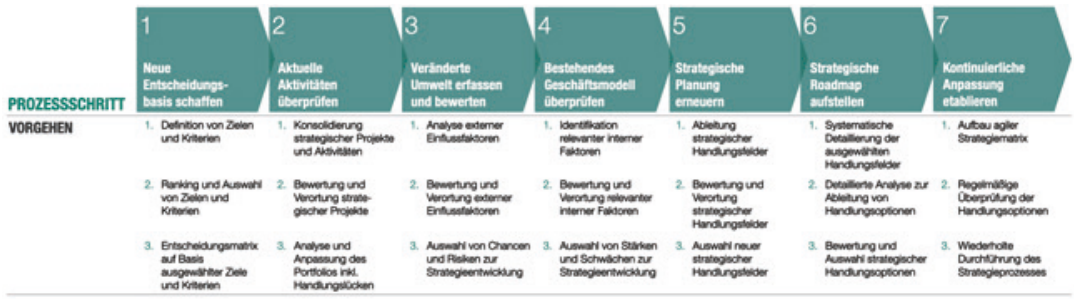

Abb. 2.31 Prozess zur Strategieentwicklung in Krisenzeiten (Gesamt) 